\title{
3 Das Konzept des Kompilationsnetzwerks
}

Die bestehenden Konzepte von Autorschaft werden der Textproduktion frühneuzeitlicher Rezeptkompilationen nicht gerecht. Dies zum einen, weil sie von einem Schreiben von neu Ausgedachtem ausgehen und zum anderen, weil sie den materiellen Aspekt - also die Bücher als Grundlage neuer Bücher - nicht miteinbeziehen. Aus diesem Grund schlage ich das Konzept des Kompilationsnetzwerks vor. Es geht davon aus, dass mehrere Akteurinnen und Akteure in die Produktion eines Textes involviert waren und sind und berücksichtigt auch Bücher in ihrer Materialität als Teil der Autorschaft. Die Vorstellung des Kompilationsnetzwerks wurde in einem immer wieder spiegelnden Verfahren zwischen Empirie und Theorie entwickelt. Die empirische Materialbasis bildeten Korrespondenzen und Publikationen, die von Johann Jacob Wecker verfasst oder unter seinem Namen erschienen sind. Methodologisch ist das Konzept beeinflusst von Ideen der Mikrogeschichte, der Buchwissenschaft und der Praxistheorie. Da auf die Mikrogeschichte bereits in Kapitel 1.3 eingegangen wurde, wird hier auf die beiden letztgenannten Bereiche fokussiert.

\subsection{Buchwissenschaftliche Modelle}

Die Buchwissenschaft befasst sich mit dem Buch, wobei es unzählige Vorstellungen davon gibt, was ein Buch sein soll. ${ }^{347}$ Neben dieser Terminologie gibt es unzählige Vorstellungen davon, was ein Buch sein soll. Mit dieser Frage hat sich schon Immanuel Kant 1796 auseinandergesetzt. Für ihn ist ein Buch „eine Schrift, (ob mit der Feder oder durch Typen, auf wenig oder viel Blăttern verzeichnet, ist hier gleichgůltig) welche eine Rede vorstellt, die jemand durch sichtbare Sprachzeichen an das Publikum hålt. “348 Bei dieser Definition spielt es keine Rolle, ob ein Text handschriftlich verfasst oder gedruckt wurde - wichtig ist das Adressieren an ein Publikum. Die Ansicht, dass Bücher nicht unbedingt gedruckt sein müssen, wird auch heute noch vertreten. So ist ein Buch laut Ursula Rautenberg ein „Überlieferungsträger von Texten und Bildern in handschriftlicher, gedruckter und elektronischer Form“. ${ }^{349}$ Folglich können auch Papyrusrollen

347 Vgl. Howsam: Old Books and New Histories, 2006, Preface, S. VIII sowie Kapitel 1.4.2 zur Buchwissenschaft.

348 Kant, Immanuel: Werke in zwölf Bänden. Band 8. Frankfurt am Main: 1977, S. 388-406. 349 Rautenberg, Ursula: Wir lesen Bücher, nicht Texte. Der Studiengang „Buchwissenschaft“ an der Universität Erlangen, in: Kerlen, Dietrich; Kirste, Inka (Hg.): Buchwissenschaft und Buch-

Ә OpenAccess. (C) 2022 Simone Zweifel, publiziert von De Gruyter. (cc))BY Dieses Werk ist lizenziert unter einer Creative Commons Namensnennung 4.0 International Lizenz. https://doi.org/10.1515/9783110740516-004 
sowie digitalisierter Text Buch sein. ${ }^{350}$ Wichtig ist jedoch immer die Verbindung des Textes mit einem Medium. Dies betont auch Roger Chartier, für den die Materialität des Buches nicht von jener des Textes getrennt werden kann, unabhängig davon, ob es sich um gedruckten oder handgeschriebenen Text handelt. ${ }^{351}$ Damit sind die beiden Formen nicht gegensätzlich, und somit kein Ausdruck einer getrennten „scribal culture“ und „print culture“, wie es insbesondere Elizabeth Eisenstein formuliert hat. ${ }^{352}$ Vielmehr gehen Chartier und andere davon aus, dass die handschriftliche Buchpublikation auch nach der Erfindung des Buchdrucks bedeutsam war. ${ }^{353}$ Dies zeigen unter anderem frühneuzeitliche Rezeptbücher, bei denen Druck und Manuskript co-existierten und sich gegenseitig beeinflussten. ${ }^{354}$ Dass beide als Bücher betrachtet werden können, ist meines Erachtens auch dadurch zu begründen, dass sie auf die gleiche Art und Weise rezipiert werden können: sowohl handschriftliche als auch gedruckte Texte in Rezeptbüchern werden durch Blättern von Seiten gelesen. Dabei können einzelne Seiten übersprungen werden, die Seiten können durch Einlageblätter oder Ähnliches markiert werden, so dass die zuletzt gelesene Stelle oder das Lieblingsrezept rasch wiedergefunden werden kann. Dies unterscheidet neben der anderen Materialität einen Text im Codex von solchen auf Pergamentrollen oder aber von digitalen Texten.

Ein Buch ist jedoch nicht nur ein Artefakt, in dem Text materialisiert ist, es ist auch ,both the product of one complex set of social and technological processes and also the starting point for another". ${ }^{355}$ In diese Prozesse involviert waren nach Adrian Johns Menschen, Maschinen und Materialien, die zusammen agieren mussten, um Bücher herstellen $\mathrm{zu}$ können. ${ }^{356}$ Damit teilt er eine wichtige

wirkungsforschung. VIII. Leipziger Hochschultage für Medien und Kommunikation. Leipzig: Institut für Kommunikations- und Medienwissenschaft, 2000, S. 31-42, hier S. 32.

350 Ebd. Dem stimmt auch Leslie Howsam zu: Dies.: The Study of Book History, 2015, S. 2 f. 351 Chartier: La main de l'auteur et l'esprit de l'imprimeur, 2015, S. $14 \mathrm{f}$.

352 Eisenstein: The Printing Press as an Agent of Change, 2005; Eisenstein: The Printing Revolution in Early Modern Europe, 1993. Gegen diese Trennung hat auch Adrian Johns argumentiert: Ders.: The Nature of the Book, 1998, Introduction.

353 U.a. Chartier: La main de l'auteur et l'esprit de l'imprimeur, 2015, S. 23; Johns: The Nature of the Book, 1998; Leong: Recipes and Everyday Knowledge, 2018; Ezell, Margaret J.M.: Manuscript and Print Cultures 1500 -1700, in: Berensmeyer, Ingo; Buelens, Gert; Demoor, Marysa (Hg.): The Cambridge Handbook of Literary Authorship. Cambridge: Cambridge University Press, 2019, S. $115-132$.

354 Leong: Recipes and Everyday Knowledge, 2018.

355 Johns: The Nature of the Book, 1998, S. 3.

356 Ebd. 
Grundannahme dieses Buches: dass unterschiedliche Akteurinnen, Akteure und Elemente interagieren mussten, um Bücher zu kreieren.

Auf den sozialen Aspekt des Buches hat Donald F. McKenzie in seiner Bibliography and the Sociology of Texts aufmerksam gemacht. ${ }^{357}$ Er verweist auf die Sozialität von Text und darauf, dass auch die Form und Materialität den Text beeinflusst. $^{358}$ Text definiert er wie folgt:

I define 'texts' to include verbal, visual, oral, and numeric data, in the form of maps, prints, and music, of archives of recorded sound, of films, videos, and any computer-stored information, everything in fact from epigraphy to the latest forms of discography. ${ }^{359}$

Damit ist Text etwas mit einem Medium Verbundenes. Mit McKenzie gehe ich demnach nicht davon aus, dass ,letztlich alles, oder doch zumindest jedes kulturelle System und jede kulturelle Struktur, Text sein soll“, wie dies etwa von der Kultursemiotik angenommen wird. ${ }^{360}$

Kompilationstext ist von einer starken Intertextualität geprägt. ${ }^{361}$ Er ist meines Erachtens, wie bereits angesprochen, nicht fest, sondern fluide: Der Text wird immer wieder erweitert, verändert und in neuen Büchern vorübergehend fixiert. Die Gebundenheit bleibt jedoch von kurzer Dauer, da Leserinnen und Leser dem Text Unterschiedliches entnehmen; sie entwickeln ihn durch ihre Rezeption weiter. $^{362}$ Im Kontext der De Secretis waren die Bücher zudem Voraussetzung für die Entstehung neuer Bücher: Sie wurden übersetzt, aus ihnen wurde kompiliert oder Teile von ihnen wurden entnommen, um daraus neue Bücher zu produzieren. In diese Buchproduktion waren unterschiedliche Personen und Elemente involviert, weshalb diese Bücher auch als Produkte sozialer Praktiken verstanden werden können.

Damit wird erneut auf Donald F. McKenzie Bezug genommen. Nach ihm sollen bei der Analyse von Texten nicht nur die Zeichen berücksichtigt werden, sondern auch deren Komposition, die Form und das formale Design, die Verbreitung von

357 McKenzie: Bibliography and the Sociology of Texts, 2004.

358 Ebd.

359 Ebd., S. 13.

360 Pfister, Manfred: I. Konzepte der Intertextualität, in: Broich, Ulrich; Pfister, Manfred; Schulte-Middelich, Bernd (Hg.): Intertextualität. Formen, Funktionen, anglistische Fallstudien (= Konzepte der Sprach- und Literaturwissenschaft). Tübingen: De Gruyter, 1985, S.1-47, hier S. 7. 361 Der Begriff der Intertextualität ist auf Gérard Genette zurückzuführen: Genette, Gérard: Palimpsestes. La littérature au second degré (= Collection Poétique). Paris: Ed. du Seuil, 1982; Genette, Gérard: Paratexte. Frankfurt am Main: Campus Verlag, Paris: Éditions de la Maison des Sciences de l'Homme, 1989.

362 Vgl. Chartier: La main de l'auteur et l'esprit de l'imprimeur, 2015. 
Texten durch Schreiber, Buchdrucker und Verleger sowie durch verschiedene Gesellschaften, deren Sammlung und Klassifikation durch Bibliothekarinnen und Bibliothekare, die Meinung von Lesenden sowie „cognitive regenerations“ derselben. ${ }^{363}$ All diese Elemente waren Produkte respektive Teil sozialer Praktiken, also von Buchpraktiken, wie sie auch in diesem Buch analysiert werden. ${ }^{364} \mathrm{Im}$ Gegensatz zu McKenzie stehen hier jedoch die Praktiken, die hinter der Textproduktion stehen, im Vordergrund. Dazu zählt unter anderem das Beschaffen von Text sowie das Organisieren und Ordnen von Wissen. Dies sind Praktiken, die zur Komposition von Kompilationen erforderlich sind. Nicht untersucht werden hingegen die „cognititive regenerations“ der Lesenden. ${ }^{365}$ Diese zu analysieren wäre zwar höchst spannend, ist jedoch in Bezug auf den hier zu erforschenden Kontext nicht realisierbar. Denn dafür müsste man die Lesenden befragen, was bei Personen aus dem 16. Jahrhundert schlicht nicht umsetzbar ist.

Die Elemente der Textproduktion, die McKenzie beschreibt, finden sich teilweise auch im Kommunikationskreislauf Robert Darntons, einem Modell, das den Kreislauf der Buchproduktion darstellt (Abb.6):

[...] [it, SZ] runs from the author to the publisher (if the bookseller does not assume that role), the printer, the shipper, the bookseller, and the reader. The reader completes the circuit, because he influences the author both before and after the act of composition. Authors are readers themselves. By reading and associating with other readers and writers, they form notions of genre and style and a general sense of the literary enterprise, which affects their texts $\left[\ldots . . . .^{366}\right.$

Darnton's Modell stellt einen vollen Kreislauf dar, in den verschiedene Menschen involviert sind, die wiederum in einen Kontext eingebunden sind. ${ }^{367}$

In diesem Modell wird deutlich, dass die Autorinnen und Autoren und die Verlegerinnen und Verleger nicht alleine stehen, sondern dass sie Teil eines Geflechts von Akteurinnen und Akteuren sind. In der Mitte dieses Kreislaufs finden sich „intellecutal influences and publicity“, „political and legal sanctions“, „economical and social conjuncture“. Man könnte diese auch als kulturelle Kontexte bezeichnen, in denen die Text- und Buchproduktion eingebunden ist. ${ }^{368}$ Der Darntonsche Kommunikationskreislauf benennt folglich viele Elemente, die

363 McKenzie: Bibliography and the Sociology of Texts, 2004, S. 12.

364 Vgl. ebd. Zu Praktiken siehe u. a. Reckwitz: Toward a Theory of Social Practices, 2002, S. 243 263 sowie Kapitel 3.2 zur Praxistheorie.

365 McKenzie: Bibliography and the Sociology of Texts, 2004, S. 12.

366 Darnton: What is the History of Books?, 1982, S. 67.

367 Ebd., S. 68.

368 Ebd. 


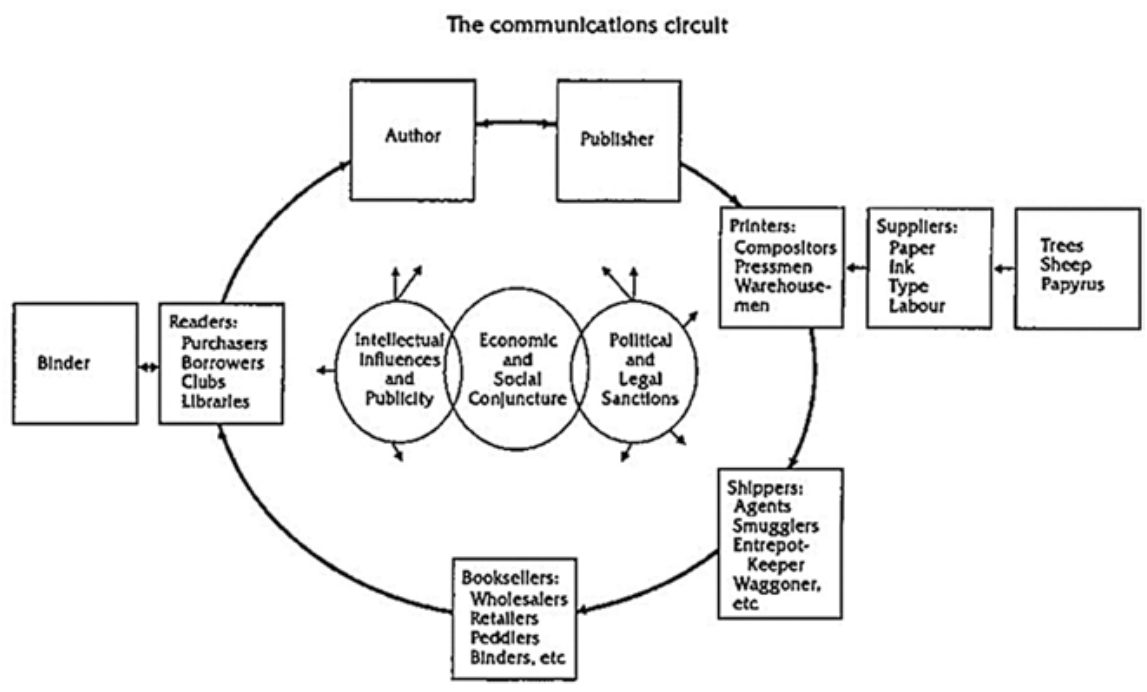

Abbildung 6: Robert Darntons Kommunikationskreislauf.

auch für das Kompilationsnetzwerk von Bedeutung sind, da sie aktiv die Buchproduktion beeinflussen. Beispiele dafür sind etwa die Autorinnen und Autoren, Druckerinnen und Drucker, Agentinnen und Agenten sowie die Lesenden. Diese bildeten in der Vorstellung des Kompilationsnetzwerks jedoch keinen Kreis, sondern ein Geflecht, da sie auch mehrfach miteinander verbunden sein konnten.

Der Darntonsche Kommunikationskreislauf wurde in der Forschung unter anderem dafür kritisiert, dass es sehr androzentriert sei und die Funktion von Frauen im frühneuzeitlichen Buchdruck vernachlässige. Sie waren oftmals eine Art „hidden players“ in der Buchproduktion. ${ }^{369}$ Man könnte auch sagen, dass sie ein Teil des Geflechts der Buchproduktion sind, der - aufgrund der fehlenden Sichtbarkeit - in diesem Modell verloren geht. Betrachtet man die Autorschaft als Kompilationsnetzwerk, so werden auch Frauen, deren Namen nicht auf dem Titelblatt stehen, aber die in die Buchproduktion involviert waren, Teil der Autorschaft.

Thomas R. Adams und Nicolas Barker bemängeln am Darntonschen Modell, dass es sich mit den Menschen beschäftige, die mit Büchern handelten und diese produzierten, und weniger mit den Büchern selbst. Es befasse sich vorwiegend mit

369 Vgl. Smith: Grossly Material Things, 2012, Introduction, insbesondere S. 6. Zur aktiven Rolle von Frauen im Buchdruck siehe Coldiron: Women in Early English Print Culture, 2010, insbesondere S. 72-77; Smith: Grossly Material Things, 2012. 
der Geschichte der Kommunikation und vernachlässige unter anderem die Unplanbarkeit und Zufälligkeit des Buchmarktes. ${ }^{370}$ Aus diesem Grund nahmen die beiden einen Richtungswechsel vor und entwickelten ein neues Modell, das stark geprägt war von jenem Darntons. Das Modell von Adams und Barker stellt nicht die Menschen, sondern die einzelnen Produktionsschritte in den Vordergrund (Abb.7):

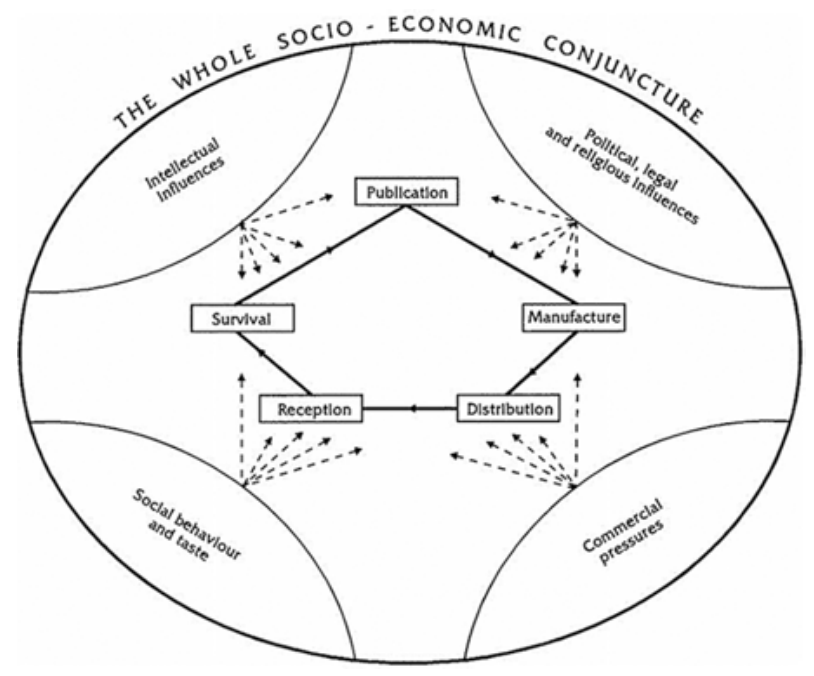

Abbildung 7: Das Buchproduktionsmodell von Thomas R. Adams und Nicolas Barker.

Als Schritte werden die Herstellung, Verteilung, Distribution, der Fortbestand sowie die Publikation von Büchern genannt. Diese sind beeinflusst von äusseren Faktoren, wie dies schon Darnton aufgezeigt hat, und bilden ein Kommunikationssystem. ${ }^{371}$ Auf den ersten Blick könnte man meinen, dass die Akteurinnen und Akteure der Buchproduktion, die bei Darnton so wichtig sind, bei Adams und Barker verschwinden. Dem ist jedoch nicht so; denn nach ihnen beginnt der Buchproduktionsprozess mit der Entscheidung von ,author, patron (or financier), manufacturer and distributor“, ein Buch herzustellen. ${ }^{372}$ Damit sind sie die wichtigsten Akteure oder Akteurinnen in diesem Prozess. Sie bestimmen nach Adams und Barker über die Erschaffung oder Nicht-Erschaffung eines Buches, wobei sowohl kreative und kommunikative Aspekte als auch der Profit sowie der

370 Adams, Barker: A New Model for the Study of the Book, 1993, S. 12.

371 Ebd., S. $14 \mathrm{f}$.

372 Ebd., S. 16. 
Fortbestand berücksichtigt würden. ${ }^{373}$ Damit wird diesen Menschen eine grosse Bedeutung zugemessen, obwohl sie im Modell nicht zu finden sind. Das Modell fokussiert auf fünf Schritte der Buchproduktion, auf die an dieser Stelle eingegangen werden soll, um einen besseren Einblick in dasselbe zu ermöglichen. Dies ist deshalb wichtig, weil Adams und Barker in ihrem Modell viele Praktiken darstellen, die in dieser Studie als Buchpraktiken von Relevanz sind.

Für Adams und Barker stellt der erste Schritt zu einer Publikation demnach nicht die Schaffung eines Textes, sondern die Entscheidung, etwas zu veröffentlichen, dar. ${ }^{374}$ Zwar würde der Autor oder die Autorin diese Entscheidung beeinflussen - mit einem Text allein gibt es nach Adams und Barker jedoch noch kein gedrucktes Buch. ${ }^{375}$ Dies gilt sowohl für die Frühe Neuzeit als auch für die Moderne, was die grosse Anzahl nicht veröffentlichter Romane zeigt, die regelmässig an die Verlagshäuser geschickt werden. Teil dieses Entscheidungsprozesses ist nach Adams und Barker auch jene für ein Format. ${ }^{376}$ Geht es bezüglich der Frühen Neuzeit vor allem um die Grösse, aber auch die Qualität des Papieres, so stellt sich heute die Frage nach Hard-Cover oder Taschenbuch oder aber nach einer digitalen Publikation. Auch in der Frühen Neuzeit war die Frage nach dem Format eine bedeutende: Druckte man in Folio, war das Buch repräsentativer; ein Duodecim konnte man dafür einfacher herumtragen. Ein Beispiel dafür ist die Practica medicinae generalis von Johann Jacob Wecker: Das kleine Büchlein, das Wissen zur Medizin, aber auch zu Medikamenten enthielt, konnte problemlos in einer Tasche zu einem Krankenbesuch mitgenommen werden. ${ }^{377}$ Neben den von Adams und Barker genannten Faktoren spielen meines Erachtens auch weitere Orientierungspunkte - wie etwa die Frage nach dem Genre oder dem erhofften Publikum - in diesem Stadium der Erschaffung eines Buches eine Rolle.

Der nächste Schritt nach Adams und Barker ist das „Manufacturing“, womit der technologische Prozess inklusive der für den Druck benötigten Dinge, zu denen die Druckerpresse, aber auch Papier und Tinte zu zählen sind, gemeint ist.

\section{Ebd.}

374 Ebd. In diesen Entscheidungsprozess waren in der Frühen Neuzeit oft mehrere Akteure eventuell auch Akteurinnen - involviert. Siehe Kapitel 4 zu Buchpraktiken sowie Kooistra, Milton: To Print or Not to Print. The Influence of Humanist Networks on the Publishing Programme of Printers in and around Basel in the early Sixteenth Century, in: Christ-von Wedel, Christine; Grosse, Sven; Hamm, Berndt (Hg.): Basel als Zentrum des geistigen Austauschs in der frühen Reformationszeit (= Spätmittelalter, Humanismus, Reformation). Tübingen: Mohr Siebeck, 2014, S. 135-146, hier S. 135-137.

375 Adams, Barker: A New Model for the Study of the Book, 1993, S. 18.

376 Ebd.

377 Siehe z.B. Wecker, Johann Jacob: Practica medicinae generalis. 'A Io. Iacobo VVeckero Poliatro Colmariense VII. libris explicata. Basileae: Hieron. Frobum, \& eius affinem, 1585. 
Sie können, wie bereits angesprochen, den ersten Schritt des Entstehungsprozesses beeinflussen. ${ }^{378}$ In die Organisation und Herstellung dieser Produkte waren viele Personen involviert, wobei sich Adams und Parker weniger für diese interessieren als für den Buchproduktionsprozess. ${ }^{379}$ Dabei spielen Dinge, die für den Druck benötigt werden, eine Rolle, wobei im Kontext von Kompilationen insbesondere Bücher zu diesen Dingen zu zählen sind. ${ }^{380}$ Sie könnten theoretisch in diesem Modell mitgedacht sein, was jedoch nirgendwo ersichtlich wird. Es scheint nämlich, dass hier die für das Buch und nicht für den Text gefragten Materialien gemeint sind. Damit unterscheide ich Text und Buch, wobei ich das Buch als materialisierten Text verstehe und als ein $\mathrm{zu}$ einem spezifischen Zeitpunkt fixiertes Resultat von Buchpraktiken. ${ }^{381} \mathrm{Zu}$ diesem Zeitpunkt verfestigt sich der Text, der zum Teil des Buches wird. ${ }^{382}$ Text und Buch sind nur so lange fixiert, bis Rezipierende diese durch ihre Lektüre wieder verändern und weiterentwickeln. ${ }^{383}$

Auf das „Manufacturing“ folgt die Distribution. Unter diesem Begriff fassen Adams und Barker die Bewegung der Bücher vom Druck bis in den Rare-BookRoom. Hier könnte man die digitalisierten Ausgaben ergänzen, durch die sich die Bücher in einer anderen Medialität weiterbewegen. Auch die Frage nach dem Interesse, ein Buch zu besitzen, wird diesem Bereich zugerechnet, wie auch die Anzahl der Neuauflagen oder aber das Fehlen von Neuauflagen. ${ }^{384}$

Ein weiterer Schritt ist jener der Rezeption. Adams und Barker betonen, dass sie häufig keine Spuren hinterlasse und dass deren Verständnis, obwohl schwierig zu erlangen, äusserst wichtig sei, wenn man den Impact eines Buches evaluieren wolle. ${ }^{385}$ Obwohl die Anzahl der Neudrucke einen Anhaltspunkt bezüglich der Popularität eines Werkes gebe, so seien Fragen wie jene nach den tatsächlich gelesenen Büchern sowie jene, welche Bereiche der Leserschaft sie erreichten oder aber nicht, wichtiger. ${ }^{386}$ Damit werden weitere Beteiligte der Buchproduktion angesprochen: Die Lesenden. Sie haben in der Buchwissenschaft - auch in Bezug

378 Adams, Barker: A New Model for the Study of the Book, 1993, S. $18 \mathrm{f}$.

379 Vgl. ebd., S. 22.

380 Ebd., S. 18 f.

381 Damit verstehe ich den Text als Resultat von Handlungen, das wiederum Handlungen beeinflussen kann und nicht als Handlung selbst, wie dies von Monika Mommertz vorgeschlagen wird. Mommertz, Monika: „Ich, Lisa Thielen.“ Text als Handlung und als sprachliche Struktur ein methodischer Vorschlag, in: Historische Anthorpologie 4 (3), 1996, S. 303-329, hier S. 304.

382 Vgl. Bland: Early Printed Books and Manuscripts, 2010, S. 18.

383 Vgl. Chartier: La main de l'auteur et l'esprit de l'imprimeur, 2015, S. 17, $24 \mathrm{f}$.

384 Adams, Barker: A New Model for the Study of the Book, 1993, S. 22-26, 27.

385 Ebd., S. 27.

386 Ebd., S. 28. 
auf Rezeptbücher - in den letzteren Jahren an Bedeutung gewonnen. ${ }^{387}$ Sie konnten den am Ende gedruckten Text ebenfalls beeinflussen, etwa, indem sie gewisse Texte oder Formate bevorzugten. Lesen war zudem eine wichtige Buchpraktik bei der Herstellung von Büchern: Sie war notwendig, um Textstellen selektionieren zu können, die in die Texte neuer Bücher einflossen. Zur Rezeption wird bei Adams und Barker nicht nur die direkte Rezeption der Lesenden gezählt, sondern auch die Übersetzung sowie die Frage, inwiefern intendierte und tatsächliche Rezipierenden übereinstimmten sowie „the way that the ideas, and even the actual wording of those ideas, are picked up and used with or without acknowledgment by later writers for a variety of purposes, some have nothing to do with the original intention of the author and publisher." ${ }^{388}$ Dass Textmaterial auch nach dem Tod eines Autors, einer Autorin oder Druckers respektive einer Druckerin weiter verbreitet wurde, ist eine Praxis, die man in der Frühen Neuzeit häufig findet. Dies gilt auch für die Publikationen Johann Jacob Weckers: auch sie wurden nach seinem Ableben weiter gedruckt und verbreitet. Diese Rezeption dauerte bis ins 18. Jahrhundert an, was auf den grossen Erfolg der Weckerschen Publikationstätigkeit hinweist. ${ }^{389}$ In diesem Zusammenhang ist nicht nur die Frage nach der Rezeption von Interesse, sondern auch jene der Autorisierung der Wissensbestände. Denn das von den Weckerschen Kompilationsnetzwerken gesammelte und kompilierte Wissen wurde später als Weckersches Wissen tradiert. $^{390}$

„Survival“ ist der nächste Schritt im Modell von Adams und Barker. Die beiden gehen davon aus, dass populäre Publikationen öfters neu aufgelegt werden, diese aber aufgrund starken Gebrauchs weniger häufig überliefert werden. ${ }^{391}$ Dies trifft auf Bücher des 16. Jahrhunderts nicht unbedingt zu, da eine hohe Anzahl an

387 Siehe u. a. Chartier: The Order of Books, 1994; Ders.: Lesewelten. Buch und Lektüre in der frühen Neuzeit (= Historische Studien, Bd. 1). Frankfurt am Main, New York: Campus Verlag; Paris: Ed. De la Maison des Sciences de l'Homme, 1990; DiMeo, Michelle; Pennell, Sara (Hg.): Reading and Writing Recipe Books, 1550 - 1800. Manchester: Manchester University Press, 2013; Smith, Helen: ,More swete vnto the eare / than holsome for ye mynde': Embodying Early Modern Women's Reading, in: Huntington Library Quarterly 73 (3), 2010, S. 413-432; Blair: Reading Strategies for Coping With Information Overload ca. 1550-1700, 2003; Eamon: How to Read a Book of Secrets, 2011; Jardine, Lisa; Grafton, Anthony: „Studied for Action“. How Gabriel Harvey Read his Livy, in: Past and Present 129 (1), 1990, S. $30-78$.

388 Adams, Barker: A New Model for the Study of the Book, 1993, S. 30.

389 Wecker, Zwinger: De Secretis Libri XVII, 1750.

390 Vgl. Murhard, Friedrich Wilhelm August: Geschichte der Naturlehre. Die wichtigsten Lehren der Physik. Erster Band. Historisch bearbeitet (= Bd.1, Bd. 8). Göttingen: Rosenbusch, 1799, S. $34 \mathrm{f}$. 391 Adams, Barker: A New Model for the Study of the Book, 1993, hier S. 33, $38 \mathrm{f}$. 
Drucken auch zu einer höheren Wahrscheinlichkeit der Überlieferung führte, trotz Gebrauchs. Zumindest gilt dies für die Weckerschen Drucke, von denen viele Gebrauchsspuren aufweisen; dennoch sind sie in vielen Bibliotheken zu finden und können noch immer online erworben werden. ${ }^{392}$ Vom „Survival“ kann ein Buch wiederum in eine neue „Publication“ übergehen und der Kreislauf beginnt von Neuem. ${ }^{393}$

Die von Adams und Barker wie auch die von Robert Darnton beschriebenen Schritte zeigen wichtige Stationen der Buchproduktion auf. Obwohl die Modelle für die Moderne entwickelt wurden, sind sie auf die Frühe Neuzeit übertragbar. Was die beiden Modelle deutlich machen - und was sie als Hintergrundmodelle des Kompilationsnetzwerks bedeutsam macht - ist, dass mehrere Personen in die Publikation von Büchern involviert waren und sind, dass die Publikationstätigkeit mehrere Schritte umfasst und dass auch kulturelle, politische und soziale Faktoren die Entstehung von Büchern beeinflussen konnten und können. Sie zeigen jedoch nicht, wie diese äusseren Faktoren mit den Akteurinnen und Akteuren zusammenhängen oder -hingen, was hier thematisiert werden soll. Beide Modelle befassen sich zudem mit der Herstellung von Büchern - nicht aber mit der dahinterstehenden Produktion von Text. Letztere ist zwar mit ersteren verknüpft; dennoch sind und waren darin weitere Personen, Elemente sowie Praktiken eingebunden, die in den beschriebenen Modellen nicht berücksichtigt sind. Diese Erkenntnis wird nur dank des Zoomens hinter den Text möglich. ${ }^{394}$ Es belegt, dass weitere Praktiken, Personen und Elemente bedeutsam waren und sind, die in den hier angesprochenen Modellen nicht auftauchen. Bezüglich der frühneuzeitlichen Kompilationsproduktion sind insbesondere das Korrespondieren und Beschaffen von Büchern mit den darin involvierten Personen, wie etwa Botinnen und Boten, sowie die Bücher selbst als Elemente der Textproduktion zu nennen.

Demnach trenne ich die Text- von der Buchproduktion, wobei ich, wie bereits angesprochen, davon ausgehe, dass sich der Text zum Zeitpunkt des Druckes verfestigt und zum Teil des Buches wird. ${ }^{395}$ Dass für die hier untersuchten Akteurinnen und Akteure die Buchproduktion mit dem Druck eines Buches nicht beendet war und dieses auch nicht zwingend als „fertig“ betrachtet wurde, illustriert folgendes Zitat aus einem Brief Johann Jacob Weckers an Theodor Zwinger:

392 Siehe z.B. ViaLibri. Online unter: https://www.vialibri.net/searches/201905201106dAn Mxcufq [24.02.2021].

393 Adams, Barker: A New Model for the Study of the Book, 1993, hier S. 33, $38 \mathrm{f}$.

394 Zum Zoomen siehe Pomata: Close-Ups and Long Shots, 1998; Kracauer: History. The Last Things Before the Last, 1969.

395 Vgl. Bland: Early Printed Books and Manuscripts, 2010, S. 18. 
Ich möchte gerne erfahren, wie es um meine Tafeln steht, ob man anfange, sie zu drucken, oder wie sie Euch gefallen. Ich kann wohl erkennen und weiss wohl, dass sie stark verbessert werden sollten. Aber für dieses Mal muss man es so belassen, bis ich einmal mehr Zeit und eine bessere Gelegenheit haben mag. ${ }^{396}$

Hier wird betont, dass man die Textproduktion für dieses Mal so stehen lassen müsse. Der Verweis auf „dieses Mal“ macht deutlich, dass Wecker von einem anderen Mal ausging, also von einer zukünftigen Gelegenheit, den Text zu verbessern. Dies zeigt, dass der Text nicht als etwas Fertiges betrachtet wurde, sondern als Etwas, an dem man weiterarbeiten konnte und das daraufhin erneut publiziert werden konnte. Diese Aussage zeigt, dass Neuauflagen bei der Produktion eines Buches zum Teil bereits mitgedacht wurden: ein Buch konnte immer wieder verbessert werden. ${ }^{397}$ Im Kontext der Kompilationen kommt zur Verbesserung die Erweiterung dazu: nur durch diese war es möglich, so viele - auch zeitgenössische - Autorinnen und Autoren wie möglich zu versammeln, was bei Kompilationen bedeutsam war. ${ }^{398}$

Der Text veränderte sich demzufolge immer wieder und wurde durch jeden Druck neu fixiert und materialisiert. Dies nicht nur in unterschiedlichen Ausgaben, sondern auch in verschiedenen Sprachen: die Publikationen Weckers wurden sowohl ins Französische als auch ins Englische übersetzt. ${ }^{399}$ Die Vorstellung, dass es sich bei Editionen um jeweils neue Drucke - und nicht etwa ein Werk handelt, findet sich auch bei Roger Chartier: „La ,même‘ œuvre, en effet, n’est plus la même quand changent sa langue, sa ponctuation, son format ou sa mise en page. ${ }^{“ 400}$ Dies gilt auch für die Weckerschen Publikationen. Sie konnten sich, trotz zum Teil gleichen Titeln, formell und inhaltlich unterscheiden und werden

396 „Wie eß vmb meine tabulas stende, ob man sie anfache zů trucken, oder wie sie euch gefallen, mechte ich wol vernemmen. Kann wol erkennen, vnd weiß wol, dz sie viel verbesseret mechten werden. Aber man můß aůff diß mal für gůtt haben, biß ich ein mal eher zeitt vnd besser gelegenheitt haben mag.“ Wecker, Johann Jacob: Brief an Theodor Zwinger. UB Basel, Frey-Gryn Mscr II 4:Bl.315.

397 Ein ähnliches Phänomen stellt Benito Rial Costas bei frühneuzeitlichen Brevarien fest: Rial Costas, Benito: International Publishing and Local Needs: the Breviaries and Missals Printed by Plantin for the Spanish Crown, in: McLean, Matthew; Barker, Sara (Hg.): International Exchange in the Early Modern Book World (= The Handpress World). Leiden, Boston: Brill, 2016, S. 15-30, hier S. 20.

398 Siehe dazu z. B. Wecker, Johann Jacob: Brief an Theodor Zwinger. UB Basel, Frey-Gryn Mscr II 4:Nr. 326.

399 U.a.Wecker, Johann Jacob: Les secrets et merveilles de natvre. Receuillis de diuers Autheurs, \& Diuisez en XVII. liures [...]. Vaze d'or: Barthelemi Honorati, 1586; Wecker, Read: Eighteen Books of the Secrets of Art \& Nature, 1660.

400 Chartier: La main de l'auteur et l'esprit de l'imprimeur, 2015, S. 15. 
hier als einzelne Materialisierungen von Text zu jeweils einem Buch verstanden. Doch nicht nur die einzelnen Drucke differieren: die Texte veränderten sich auch durch die Lesenden, etwa, indem sie neue Rezepte von Hand einfügten. ${ }^{401}$

Um Text und auch Bücher herstellen zu können, mussten verschiedene Praktiken ausgeführt werden. Auf einige dieser Praktiken, wie etwa das Publizieren, Distribuieren und die Rezeption von Büchern haben unter anderem Adams und Barker verwiesen. ${ }^{402}$ Im Kompilationsnetzwerk kommen jedoch weitere Praktiken dazu: das Sammeln von Textbestandteilen, aus denen neuer Text entwickelt werden konnte, das Korrespondieren und Beschaffen von Büchern, das Lesen, das Exzerpieren, das Selektionieren, das Ordnen, das Zusammenfügen sowie das Korrigieren von Textmaterial. ${ }^{403}$ Diese Praktiken werden hier als Buchpraktiken bezeichnet, also auf die Herstellung von einem Buch ausgerichtete Praktiken. In viele dieser Praktiken waren mehrere Personen involviert: Sie - und nicht etwa Wecker allein - machten die frühneuzeitliche Kompilationsproduktion erst möglich.

An den Buchpraktiken von Kompilationen waren nicht nur Menschen beteiligt: Auch Dinge, wie etwa Papier und Tinte, konnten und können die Buchproduktion beeinflussen. Dies betont Donald F. McKenzie:

Almost all texts of any consequence are the product of the concurrent inter-action of ideologies and institutions, of writers, publishers, printers, binders, wholesalers, travellers, retailers, as well as of the material sources (and their makers and suppliers) of type, paper, cord, and all the appurtenances of a printing house..$^{404}$

Bei den „material sources“, auf die McKenzie verweist, handelt es sich um die materiellen Grundlagen, die für die Produktion eines Buches notwendig sind. Für die Produktion von Text bei Kompilationen waren weitere „material sources“ unabdingbar: die Bücher, aus denen Text kopiert wurde. Denn viele Buchpraktiken, die zu Kompilationen führten, konnten nur ausgeführt werden, wenn Menschen mit nicht-menschlichen Elementen, in diesem Falle insbesondere Büchern, verbunden waren. ${ }^{405}$ Waren sie miteinander verknüpft, so konnten Buch-

401 Zur Veränderung von Text durch die Lesenden siehe ebd., S. 15, $24 \mathrm{f}$.

402 Adams, Barker: A New Model for the Study of the Book, 1993, S. 14.

403 Teile dieser Praktiken werden auch von Ann Blair sowie von Helmut Zedelmaier berücksichtigt: Blair: Too Much to Know, 2010; Zedelmaier: Werkstätten des Wissens zwischen Renaissance und Aufklärung, 2015.

404 McKenzie: 5. The London Book Trade in 1644, 2002, hier S. 128.

405 Ann Blair nennt das Speichern, Auswählen, Ordnen und Zusammenfassen von Information als wichtige Elemente der Kompilation: Blair: Reading Strategies for Coping With Information Overload ca. 1550 -1700, 2003, S. 12. 
praktiken ausagiert werden. Praktiken sind es deshalb, weil es sich dabei um routinisierte Handlungen handelte, die von den Personen unterschiedlich agentisch orientiert vollzogen wurden. ${ }^{406}$ In diese Vorstellung von Praktiken flossen unterschiedliche Ideen aus der Praxistheorie mit ein, auf die im Folgenden eingegangen wird..$^{407}$

\subsection{Praxistheorie}

Unter Praktiken versteht Andreas Reckwitz routinisierte Typen des Verhaltens und Verstehens, die an unterschiedlichen Orten und Zeitpunkten auftreten können. Sie bestehen aus mehreren, miteinander verbundenen Elementen. $\mathrm{Zu}$ diesen Elementen zählt Reckwitz unter anderem Formen körperlicher und mentaler

406 Vgl. Reckwitz: Toward a Theory of Social Practices, 2002, S. 240; Ders.: Grundelemente einer Theorie sozialer Praktiken, in: Ders. (Hg.): Unscharfe Grenzen. Perspektiven der Kultursoziologie (= Sozialtheorie). Bielefeld: transcript, 2008, S. 97-130, S. 112; Füssel, Marian: Die Praxis der Theorie. Soziologie und Geschichtswissenschaft im Dialog, in: Brendecke, Arndt (Hg.): Praktiken der Frühen Neuzeit. Akteure - Handlungen - Artefakte (= Frühneuzeit-Impulse). Köln, Wien: De Gruyter, 2015, S. 21-33, hier S. 23.

407 Die Praxistheorie ist ein in den letzten Jahren gewachsenes Forschungsfeld, das auch starke Auswirkungen auf die Geschichtswissenschaften hatte und immer noch hat. Siehe Füssel: Die Praxis der Theorie, 2015. Wichtige Arbeiten zur Praxistheorie sind u.a. Schäfer, Hilmar (Hg.): Praxistheorie. Ein soziologisches Forschungsprogramm (= Sozialtheorie). Bielefeld: transcript, 2016; Bueger, Christian; Gadinger, Frank: International Practice Theory. New Perspectives. Basingstoke: Palgrave Macmillan, 2014; Elias, Friederike; Franz, Albrecht; Murmann, Henning u. a. (Hg.): Praxeologie. Beiträge zur interdisziplinären Reichweite praxistheoretischer Ansätze in den Geistes- und Sozialwissenschaften (= Materiale Textkulturen, Bd. 3). Berlin, Boston, Mass.: De Gruyter, 2014; Schäfer, Hilmar: Die Instabilität der Praxis. Reproduktion und Transformation des Sozialen in der Praxistheorie. Weilerswist: Velbrück, 2013; Shove, Elizabeth; Pantzar, Mika; Watson, Matt: The Dynamics of Social Practice: Everyday Life and how it Changes. Los Angeles: SAGE, 2012; Schmidt, Robert: Soziologie der Praktiken. Konzeptionelle Studien und empirische Analysen. Berlin: Suhrkamp, 2012; Reckwitz, Andreas (Hg.): Unscharfe Grenzen. Perspektiven der Kultursoziologie (= Sozialtheorie). Bielefeld: transcript, 2008; Hörning, Karl H.; Reuter, Julia (Hg.): Doing Culture. Neue Positionen zum Verhältnis von Kultur und sozialer Praxis (= Sozialtheorie). Bielefeld: transcript, 2004; Reckwitz: Toward a Theory of Social Practices, 2002; Schatzki, Theodore R.: The Site of the Social. A Philosophical Account of the Constitution of Social Life and Change. University Park, Pa.: Pennsylvania State University Press, 2002; Ders.; Knorr-Cetina, Karin; Savigny, Eike von (Hg.): The Practice Turn in Contemporary Theory. London: Routledge, 2001; Schatzki, Theodore R.: Social Practices. A Wittgensteinian Approach to Human Activity and the Social. Cambridge [etc.]: Cambridge University Press, 1996. Im Folgenden fokussiere ich auf jene Elemente der Praxistheorie, die für mein Konzept des Kompilationsnetzwerks relevant sind und nehme nicht auf alle Forschungen des Feldes Bezug. 
Aktivitäten, Dinge, den Gebrauch von Dingen, emotionale Zustände sowie gewisse Wissensbestände, die wichtig sind, um Vorgänge verstehen zu können. ${ }^{408}$ Diese Verbindung von Elementen, die sowohl Dinge, Menschen als auch „nicht fassbare Elemente“ sein können, ist auch für Elizabeth Shove und andere bedeutsam: „[...] practices emerge, persist and disappear as links between their

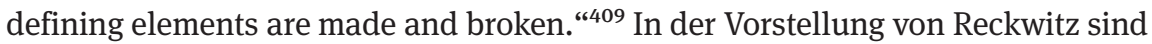
„body/mind“ die Träger von Praktiken - die einzelne Person bildet die Schnittstelle der Praktiken, die als körperlich-mentale Routine verstanden werden. ${ }^{410} \mathrm{Im}$ Gegensatz dazu nehmen Shove und andere an, dass Praktiken eine aktive Reproduktion und Performance voraussetzen: „In other words, people have to do them. “411 In beiden dieser Vorstellungen scheinen Praktiken gebunden an eine feste Struktur, innerhalb dieser Routinen ausgeführt werden. Damit betrachten sie die Praktiken aus einer Systemperspektive. ${ }^{412}$ Auch Tanja Thomas und Friedrich Krotz gehen davon aus, dass der Mensch ein ,gewordenes, habitualisiertes Wesen ist“, „das aber im Prinzip immer die Möglichkeit behält, seine Handlungsweisen $\mathrm{zu}$ hinterfragen und zu überwinden, wenn auch immer auf der Basis habitualisierter Strukturen. “413 Wie diese Strukturen überwunden werden können, bleibt jedoch offen.

Karl H. Hörning und Julia Reuter hingegen sehen Praxis, also das Zusammenkommen mehrerer Praktiken, ,als Scharnier zwischen dem Subjekt und den Strukturen angelegt. “414 Die beiden Forschenden verstehen nicht alle Handlungen als Praktiken, sondern nur jene, die sich durch „häufiges und regelmäßiges Miteinandertun“ auszeichnen. ${ }^{415}$ Doch: wie weiss man, ob eine Handlung Praktik war oder nicht? Ab wann gilt eine Handlung als regelmässig durch mehrere Menschen ausgeführt? Und: könnten nicht auch Nicht-Praktiken durch (kognitive) Routinen geprägt sein, die unbewusst beeinflussen? Es erscheint mir problematisch, zwischen Handlung und Praktik zu unterscheiden, da unklar ist, wo die Grenze gezogen werden könnte. Trotz dieser Schwierigkeit erscheint die Be-

408 Reckwitz: Toward a Theory of Social Practices, 2002, S. 240.

409 Shove, Pantzar, Watson: The Dynamics of Social Practice, 2012, S. 36.

410 Reckwitz: Toward a Theory of Social Practices, 2002, S. 256.

411 Shove, Elizabeth: Everyday Practice and the Production and Consumation of Time, in: Shove, Elizabeth; Trentmann, Frank; Wilk, Richard (Hg.): Time, Consumption and Everyday Life: Practice, Materiality and Culture. Oxford: Berg Publishers, 2009, S. 17-33, hier S. 18.

412 Vgl. Reckwitz, Andreas: Struktur. Zur sozialwissenschaftlichen Analyse von Regeln und Regelmässigkeiten. Opladen: Westdeutscher Verl., 1997, S. 39.

413 Thomas, Krotz: Medienkultur und soziales Handeln: Begriffsarbeiten zur Theorieentwicklung, 2008, S. 31.

414 Hörning, Reuter: Doing Culture: Kultur als Praxis, 2004, S. 13.

415 Ebd., S. 12. 
griffsvorstellung einer routinisierten Handlung für diese Arbeit als sinnvoll, da auch Buchpraktiken immer wieder - auch von unterschiedlichen Personen in verschiedenen Zeiten - neu ausgeführt wurden und werden.

In dieser Studie interessieren vor allem Buchpraktiken. Sie kommen dann zustande, wenn zwei Akteurinnen oder Akteure miteinander verbunden sind und können durch äussere Elemente, wie etwa zeitliche oder räumliche Kontexte, sowie durch Dinge beeinflusst werden. Denn ein Geflecht wie das Kompilationsnetzwerk, „does not have to be social only; it might as well be a set of interobjective relations.“416 Auch wenn in der Praxistheorie zum Teil zwischen Handlungen und Praktiken differenziert wird, so werden hier beide Begriffe verwendet. Dies liegt daran, dass meiner Meinung nach nicht beurteilt werden kann, ab wann eine Handlung zu einer Praktik wird und inwiefern überhaupt Handlungen existieren, die völlig frei von sind von mentalen, textuellen, kulturellen, oder wie auch immer gearteten Orientierungen respektive Strukturierungen. ${ }^{417}$

Mit „Struktur“ wird hier der Rahmen gemeint, an dem Akteurinnen und Akteure ihre Handlungen ausrichten konnten, aber nicht zwingend mussten. ${ }^{418}$ „Struktur“ verändert sich mit der Zeit, ist spatial unterschiedlich und ist entweder das Resultat routinisierter Praktiken - oder aber geht letzteren voraus. Unabhängig von der Frage, ob Praktiken diese „Struktur“ erst schaffen, oder ob das Gegenteil der Fall ist, sind Praktiken am Ende Teil einer „Struktur“, der eine gewisse Ontologie nicht abgesprochen werden kann. ${ }^{419}$ Dieser „Struktur“ inhärent sind unterschiedliche Handlungsorientierungen, die auch als agentische Orientierungen oder „agentic orientations“ bezeichnet werden können. Einen wichti-

416 Bueger, Christian; Gadinger, Frank: International Practice Theory. New Perspectives (= Palgrave pivot). Basingstoke, Hants: Palgrave Macmillan, 2015, S. 19.

$417 \mathrm{Zu}$ solchen unterschiedlichen Einflussfaktoren siehe ebd., S. 16.

418 Dazu, dass „cultural formations“ sich je nach Setting unterscheiden siehe Emirbayer, Mustafa; Goodwin, Jeff: Network Analysis, Culture, and the Problem of Agency, in: The American Journal of Sociology 99 (6), 1994, S. 1411-1454, hier S. 1442.

419 In der Forschung wird darüber diskutiert, ob Praktiken Strukturen schaffen oder umgekehrt. Dazu siehe Reckwitz: Struktur, 1997, S. 39. Diese Frage kann meines Erachtens nicht abschliessend beantwortet werden, da nicht nachgewiesen werden kann, ob zuerst Praktiken oder Strukturen existierten, weshalb sie an dieser Stelle offengelassen wird. Theodore R. Schatzki sieht die Praxistheorie als „flache Ontologie“: Ders.: Praxistheorie als flache Ontologie, in: Schäfer, Hilmar (Hg.): Praxistheorie. Ein soziologisches Forschungsprogramm (= Sozialtheorie). Bielefeld: transcript, 2016, S. 29-67. 
gen Beitrag zu solchen Orientierungen haben Ann Mische und Mustafa Emirbayer mit ihrer Studie What is Agency? verfasst. ${ }^{420}$ Sie definieren Agency als ein

[...] temporally embedded process of social engagement, informed by the past (in its habitual aspect), but also oriented toward the future (as a capacity to imagine alternative possibilities) and toward the present (as a capacity to contextualize past habits and future projects within the contingencies of the moment). ${ }^{421}$

Agency sei eingebunden in den „flow of time“. Mische und Emirbayer gehen davon aus, dass auch die strukturellen Kontexte von Handlungen temporal und relational sind und dass Akteurinnen und Akteure in diesen unterschiedliche „agentic orientations“ haben können. Je nach Kontext, in dem Handelnde agieren, würden die Möglichkeiten, selbst zu manövrieren variieren, wobei auch unterschiedliche Grade von Ideenreichtum sowie einer bewussten, überlegten Wahl vorhanden seien. ${ }^{422}$ Dementsprechend gebe es nicht eine Agency eines Akteurs oder einer Akteurin, sondern mehrere, wobei diese unterschiedlich agentisch orientiert seien. Die agentischen Orientierungen, die Emirbayer und Mische unterscheiden, sind die iterationale, die projektive und die praktisch-evaluative. ${ }^{423}$

Die iterationale Orientierung bezieht sich nach Emirbayer und Mische auf die selektive Reaktivierung bestehender, also vergangener Muster (Patterns) von Gedanken und Handlungen, die als Routinen in die praktische Aktivität inkorporiert werden. ${ }^{424}$ Solche iterationalen Elemente können auch im Netzwerk Johann Jacob Weckers festgestellt werden: Die überlieferten Briefe beispielsweise beinhalten alle eine ähnliche Struktur sowie ähnliche Formulierungen wie andere zeitgenössische Briefe. ${ }^{425}$ Die Struktur der Weckerschen Briefe kann als „past

420 Emirbayer, Mustafa; Mische, Ann: What is Agency?, in: The American Journal of Sociology 103 (4), 1998, S. 962-1023.

421 Ebd., S. 962. Siehe auch Emirbayer, Goodwin: Network Analysis, Culture, and the Problem of Agency, 1994, S. 1442f.

422 Emirbayer, Mische: What is Agency?, 1998, S. $963 \mathrm{f}$.

423 Ebd., S. 964-1000.

424 Ebd., S. 971.

425 Siehe u. a. Scherer-Boccard, Theodor; Fiala, Friedrich; Bannwart, Peter (Hg.): Archiv für die schweizerische Reformations-Geschichte, hg. auf Veranstaltung des Schweiz. Piusvereins, Band 2. Freiburg im Breisgau: Herder, 1869-1875, hier 1872, u. a. S. 339, 347, 350, 357, 374, 379, 384, 386, 390, 395, 403; Zurlaubiana AH 157/125. 1659 März 29. nach, [Zug]. Schreiben von Beat II. Zurlauben an Hans Kaspar Theobald über bestehende Differenzen im Gütertausch mit dem Kloster Frauenthal (Konzept), in: Sammlung Zurlauben. Regesten und Register zu den Acta Helvetica. Gallica, Germanica, Hispanica, Sabaudica etc. Necnon Genealogica Stemmatis Zur-Laubiani, bearb. von Stefan Hächler, Carmen Furger, Caroline Schnyder und Katrin Keller. Aarau: Sauerländer, 2010, S. 203. 
pattern“ betrachtet werden, das beim Schreiben aktiviert wird. Die einzelnen Briefelemente, zu denen Anrede, Hauptteil und Schluss zählen, werden von den Adressaten als solche erkannt und interpretiert. Die Praktiken des Briefversandes sind bei Wecker und seinen Adressaten ebenfalls routinisiert, wobei Routinen auch durchbrochen werden können und konnten, etwa dann, wenn ein Bote, durch den die Briefe transportiert werden sollten, nicht am Zielort ankam. Beispiele dafür finden sich in der Korrespondenz Johann Jacob Weckers, der etwa am 27. November 1574 an Theodor Zwinger schrieb: „Mein bott ist jetz bei 8 wůchen auss, weiss auch nitt wie ess stodt, ob er tod oder lebendig \{hab im vil gelt geben für die zerrung\}.“426 Routinen sind bedeutend für die Aufrechterhaltung der Kommunikation, die für die Kollaboration der hier zu untersuchenden Akteurinnen und Akteuren unabdingbar war. Auch die Praktik der Kompilation war ein „past pattern“, das Wecker immer wieder neu ausführte und das die Basis seiner erfolgreichen Publikationstätigkeit bildete.

Die zweite von Emirbayer und Mische beschriebene Orientierung ist die projektive, die sich auf mögliche zukünftige Handlungen bezieht. Diese würden durch Vorstellungen von Akteurinnen und Akteure generiert, wobei Hoffnungen, Ängste und Wünsche bezüglich der Zukunft zu den Orientierungspunkten zählten. Dabei könnten bestehende Denk- und Handlungsstrukturen kreativ neu konfiguriert werden. ${ }^{427}$ Die von Emirbayer und Mische angesprochenen Handlungen sind demnach auf die Zukunft ausgerichtet und beinhalten Erwartungen an dieselbe. ${ }^{428}$ Diese Ebene der Agency werde häufig zugunsten der iterationalen vernachlässigt. Die Ausrichtung auf die Zukunft erlaube einer agierenden Person, neue Handlungsmöglichkeiten zu erschliessen. Akteurinnen und Akteure, die projektiv handelten, könnten kreativer und weniger routinisiert handeln als solche, die sich iterational ausrichteten. Die Agency liegt darin, dass agierende Personen „move ,beyond themselves' into the future and construct changing images of where they think they are going, where they want to go, and how they

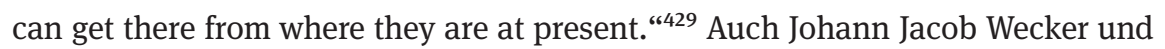
seine Kollaborierenden führten zukunftsorientierte Handlungen aus: Ein Beispiel dafür ist die Organisation und Planung neuer Bücher, bei denen sie sich etwa an künftigen Verkaufsmöglichkeiten orientierten. ${ }^{430}$

Die dritte Orientierung der Agency nach Emirbayer und Mische ist die praktisch-evaluative, die „entails the capacity of actors to make practical and nor-

426 Wecker, Johann Jacob: Brief an Theodor Zwinger. UB Basel, Frey-Gryn Mscr II 5:Nr. 94.

427 Emirbayer, Mische: What is Agency?, 1998, S. 971.

428 Ebd., S. 984.

429 Ebd., S. 983 f. Zitat: S. 984.

430 Dazu siehe Kapitel 4 zu Buchpraktiken. 
mative judgments among alternative possible trajectories of action, in response to the emerging demand, dilemmas, and ambiguities of presently evolving situations. “431 Damit sind Unsicherheiten, Konflikte und Ambiguitäten angesprochen, die Handlungen in unterschiedliche Richtungen führen können. Um eine solche Handlung in die von einem selbst gewollte Richtung zu lenken, braucht es nach Emirbayer und Mische das Bewusstsein dafür, dass die konkrete Situation auf irgendeine Art und Weise unklar, oder aber nicht eindeutig oder ungelöst ist. ${ }^{432}$ Dank eines solchen Bewusstseins könne die Handlung gesteuert werden. Folglich ermöglichen in diesem Bereich Ambiguitäten einen Handlungsspielraum, der ohne diese nicht vorhanden wäre. Solche Unsicherheiten herrschten wohl bei der Publikation der De Secretis libri XVII aus dem Jahr 1582 vor, als Pietro Perna zögerte, dieses Buch zu drucken. ${ }^{433}$ In diesem Moment hatte er unterschiedliche Entscheidungsmöglichkeiten, über die er bewusst entscheiden konnte, wobei er sich am Ende für die Publikation entschied.

$\mathrm{Zu}$ den drei besprochenen Dimensionen sollte meiner Meinung nach auch der Raum in ein Agency-Konzept miteinbezogen werden: denn die Agency eines Akteurs oder einer Akteurin kann nicht nur je nach Zeitorientierung variieren, sondern ist auch abhängig vom Raum, in dem sich die agierende Person bewegt. Es ist möglich, dass dieser Aspekt von Emirbayer und Mische bei der praktischenevaluativen Dimension mitgedacht ist; dies wird allerdings nicht explizit gemacht. Der hier gemeinte Raum muss nicht zwingend ein Gebäude, sondern kann auch ein „sozialer Raum“ sein. ${ }^{434}$ So scheint Wecker etwa in Bezug auf die Buchproduktion über eine andere Agency verfügt zu haben wie als Stadtarzt. Beide Tätigkeiten führte er gleichzeitig, aber in anderen Räumen - wohl auch in anderen „sozialen Räumen“ - aus. Der Raum ist jedoch nicht auf der gleichen Ebene wie die drei Felder Emirbayers und Misches anzusiedeln, sondern auf einer anderen, zusätzlichen, wobei auch dieses Element als relational verstanden wird.

431 Emirbayer, Mische: What is Agency?, 1998, S. 971.

432 Ebd., S. 998.

433 Wecker: De Secretis libri XVII, 1582b.

434 In der neueren Raumsoziologie wird davon ausgegangen, dass Raum ,in sozialen Relationen - vor allem durch Handeln - konstruiert wird." Eibach, Joachim: Das offene Haus. Kommunikative Praxis im sozialen Nahraum der europäischen Frühen Neuzeit, in: Zeitschrift für Historische Forschung 38 (4), 2011, S. 621-664, hier S. 640. Zur Raumsoziologie und zum „sozialen Raum“ siehe u. a. Bourdieu, Pierre: Sozialer Raum und „Klassen“. 2 Vorlesungen, Übersetzt von Bernd Schwibs (= Suhrkamp-Taschenbuch Wissenschaft, 500). Frankfurt am Main: Suhrkamp, 1985; Dünne, Jörg: Raumtheorie. Grundlagentexte aus Philosophie und Kulturwissenschaften (= Suhrkamp Taschenbücher Wissenschaft, 1800). Frankfurt am Main: Suhrkamp, 2006; Löw, Martina: Raumsoziologie (= Suhrkamp Taschenbücher Wissenschaft, 1506). Frankfurt am Main: Suhrkamp, 2001. 
Eine agierende Person kann ihre Handlung auch auf einen Raum ausrichten, ohne sich in diesem zu befinden und eine auf einen Raum bezogene Handlung kann iterational, projektiv oder praktisch-evaluativ sein. Ebenfalls wichtig erscheint mir, Alter, Gender, Herkunft, sozialer Kontext, sowie Disposition einer agierenden Person zu berücksichtigen, wenn man über Agency nachdenkt.

Weitere Orientierungspunkte respektive Einflussfaktoren auf die Agency von Akteurinnen und Akteuren sind materielle Elemente. Auf die Bedeutung von Dingen hat insbesondere Bruno Latour aufmerksam gemacht. ${ }^{435}$ Für ihn können sie auch Handelnde sein:

We don't know yet how all those actors are connected, but we can state as the new default position before the study starts that all the actors we are going to deploy might be associated in such a way that they make others do things. ${ }^{436}$

Für Latour, machen Akteurinnen und Akteure „others do things“. ${ }^{437}$ Dies können nicht nur Menschen sein, sondern auch Dinge, denen er je nach Kontext die gleiche Handlungsfähigkeit zuspricht und die bei der Handlung zu einem „Akteur-Netzwerk“ werden können. ${ }^{438}$ Dafür, dass er Dingen die gleiche Agency zuspricht wie Menschen, wurde Latour unter anderem kritisiert. ${ }^{439}$ Dieser Kritikpunkt ist meiner Ansicht nach berechtigt, da Dinge keine bewussten Entscheidungen treffen können, was ein wichtiger Punkt der Agency-Vorstellung Emirbayr und Misches ist. Demnach gehe ich davon aus, dass Dinge wie Bücher

435 Siehe u. a. Latour, Bruno: Reassembling the Social. An Introduction to Actor-Network-Theory (= Clarendon Lectures in Management Studies). Oxford: Oxford University Press, 2005; Ders.: On Actor-Network Theory. A few Clarifications, in: Soziale Welt 47, 1996, S. 369-382; Ders.: Latour, Bruno: Der Berliner Schlüssel (= Papers / WZB, Wissenschaftszentrum Berlin für Sozialforschung, 94,508). Berlin: WZB, Forschungsschwerpunkt Technik, Arbeit, Umwelt, 1994; Ders.: Where are the Missing Masses, Sociology of a few Mundane Artefacts Application, in: Bijker, Wiebe E.; Law, John (Hg.): Shaping Technology - Building Society. Studies in Sociotechnical Change (= Inside Technology). Cambridge (Mass.) [etc.]: MIT Press, 1992, S. 225-259; Ders.: Science in Action, 1987. Ich verwende einen breiten „Ding“-Begriff. Vgl. König: Das Veto der Dinge, 2012, S. 15. Zur Bedeutung von Dingen bei der Wissensproduktion siehe auch Smith, Schmidt: Smith, Introduction. Knowledge and Its Making in Early Modern Europe, 2007, S. 4; Preda: The Turn to Things, 1999. 436 Latour: Latour, Reassembling the Social, 2005, S. 107. Zu Dingen als ,aktive Entitäten“ siehe auch Preda: The Turn to Things, 1999, S. 348.

437 Latour: Latour, Reassembling the Social, 2005, S. 107. Akteurinnen und Akteure sind für ihn auch „, [...] entities that do things [...].“ Ders.: Where are the Missing Masses, 1992, S. 241.

438 Zum Akteur-Netzwerk siehe u. a. Latour: Latour, Reassembling the Social, 2005; Latour: On Actor-Network Theory. A few Clarifications, 1996.

439 U.a. Winner, Langdon: Upon Opening the Black Box and Finding It Empty. Social Constructivism and the Philosophy of Technology, in: Science, Technology \& Human Values 18 (3), 1993, S. 362-378. 
über keine Agency im handelnden Sinne verfügen; dennoch sind sie bedeutsamer Teil der Agency menschlicher Akteurinnen und Akteure. ${ }^{440}$ In diesem Kontext schreiben Jan-Hendrik Passoth, Birgit Maria Peuker und Michael W.J. Schillmeier: „Human agency is caused by and names the capacity to change structural or institutionalized relations, which comprise human and non-human entities (texts, materials, technologies, etc.). “441 Nach dieser Annahme, der ich zustimme, beeinflussen auch „non-human entities“ - im Weckerschen Falle insbesondere Bücher -, die menschliche Agency. Dies nicht in einem handelnden, sondern in einem beeinflussenden Sinne. ${ }^{442}$ Dies macht auch das Konzept der Agency von Emirbayr und Mische deutlich, insbesondere deshalb, weil es unterschiedliche Faktoren, zu denen man Dinge zählen kann, als handlungsorientierende benennt: Dinge konnten agentische Orientierungspunkte sein respektive auf diese einwirken.

Ein Beispiel für einen solchen Einfluss ist die Tatsache, dass die Handlung der buchproduzierenden Akteurinnen und Akteure vom Ziel gesteuert ist, ein Buch zu veröffentlichen. Ein Buch, aus dem durch Vorhandensein oder Nichtvorhandensein entweder kompiliert wird oder nicht, wirkt ebenfalls auf die Handlungsmöglichkeiten und die Handlungen des Geflechts ein, das ein Buch fertigstellt. Die Materialität eines Buches kann sich des Weiteren auf die temporale Orientierung der Agency auswirken. Ein konkretes Beispiel ist das folgende: Johann Jacob Wecker schrieb 1573 an Samuel Grynæus:

Was das Drucken anbelangt, wäre es mir sehr lieb, wenn ihr es noch jetzt zur kommenden Frühjahrsmesse drucken lassen würdet und dass [das Buch, SZ] bis zur nächsten Herbstmesse gedruckt und herausgegeben würde. Es sind viele, die jetzt mehr als ein ganzes Jahr

440 Dazu siehe auch Passoth, Jan-Hendrik; Peuker, Birgit Maria; Schillmeier, Michael W. J: Introduction, in: Dies. (Hg.): Agency without Actors? New Approaches to Collective Action (= Routledge Advances in Sociology). London, New York: Routledge, 2012, S. 16-26, hier S. 20. Eine ähnliche Vorstellung findet sich bei Rudolf, Florence: Questioning the human/non-human Distinction, in: Passoth, Jan-Hendrik; Peuker, Birgit Maria; Schillmeier, Michael W. J (Hg.): Agency without Actors? New Approaches to Collective Action (= Routledge Advances in Sociology). London, New York: Routledge 2012, S. 67-80, hier S. 67.

441 Passoth, Peuker, Schillmeier, Michael W. J: Introduction, 2012, S. 17.

442 Trentmann, Frank: Materiality in the Future of History: Things, Practices, and Politics, in: The Journal of British Studies 48 (02), 2009, S. 283-307, hier S. 306f. Dazu siehe auch Passoth, Peuker, Schillmeier, Michael W. J: Introduction, 2012, S. 20 und Bland: Early Printed Books and Manuscripts, 2010, S. 9. Eine ähnliche Vorstellung findet sich auch bei Rudolf: Questioning the human/ non-human distinction, 2012, S. 67. 
darauf gewartet haben. Falls ihr die Vesalischen Figuren nicht habt, muss man sie bleiben lassen und ohne dieselben drucken. ${ }^{443}$

Hier wird deutlich, dass der Inhalt des Zielproduktes Buch von demselben beeinflusst wird, dies insbesondere bezüglich des temporalen Aspektes der Frankfurter Buchmesse: Das Datum der Frankfurter Buchmesse entschied darüber, ob die Figuren Vesals Teil des Buches sein würden oder nicht. Nach meinem momentanen Erkenntnisstand ist kein Buch Weckers mit Bildern Vesals überliefert; ein Beweis für die Nichtexistenz eines solchen Buches ist dies jedoch nicht, da es auch nicht mehr vorhanden sein könnte. Im eben zitierten Brief wird zudem deutlich, dass sich der Einfluss der Messe nicht auf das am Ende produzierte Buch beschränkte, sondern sich auch direkt auf die Menschen auswirkte, die seit einem Jahr auf das Buch gewartet hätten und dieses lesen, benutzen, oder weiterverkaufen wollten. Diese potentiellen Kaufenden waren - als agentische Orientierungspunkte - ebenfalls Teil des buchproduzierenden Geflechts, des Kompilationsnetzwerks.

Dinge können demnach, so Elizabeth Shove, Mika Pantzar und Matt Watson, Elemente sein, die konnektiert werden, wenn Praktiken ausgeführt werden. ${ }^{444}$ Denn für die Forschenden entstehen Praktiken dann, wenn sich Verbindungen zwischen Elementen, die Menschen oder eben auch Dinge sein können, bilden und sie enden zu dem Zeitpunkt, bei dem sich diese Verbindungen auflösen. ${ }^{445}$ Demnach können Praktiken dann ausgeführt werden, wenn Elemente miteinander verbunden sind, wobei diese ein Geflecht bilden können. Solche Geflechte können als Netzwerke bezeichnet werden, die unterschiedliche Personen und Dinge sowie äussere Faktoren umfassen. ${ }^{446}$

443 „So vil daß trucken betriffet, were mir vast lieb, daß irß noch ietz künfftiger franckfurter vasten meß ad prelum kummen liesen, vnd auff die <ander> herbst meß getruckt vnd auß kummen mechte. Eß sindt vil, die ietz mher dan ein gantz iar darauff gewartet haben. So ir <die> Vesalij figuras nitt haben megen, můß man sie bleiben lassen, vnd on die selbigen trůcken [...].“ Wecker, Johann Jacob: Brief an Theodor Zwinger. UB Basel, G² I 30 fol. 185. 186 [Apogr.: G II 36, 1]. Bei diesem Brief handelt es sich um eine Kopie; das Original ist nicht überliefert.

444 Shove, Pantzar, Watson: The Dynamics of Social Practice, 2012, 36. Zur Bedeutung von Dingen siehe u.a. Trentmann: Materiality in the Future of History: Things, Practices, and Politics, 2009; Shove: Everyday Practice and the Production and Consumation of Time, 2009; König: Das Veto der Dinge, 2012; Preda: The Turn to Things, 1999.

445 Shove, Pantzar, Watson: The Dynamics of Social Practice, 2012, 36.

446 Unter ,äusseren Faktoren“ verstehe ich Elemente, die nicht Teil der Verbindung von zwei Elementen, deren Zusammenwirkungen zu Praktiken führen, sind, sondern die von aussen auf solche Verbindungen einwirken. 
Bei der Erforschung von Netzwerken wird zwischen zwei Ansätzen unterschieden: Einem quantitativen Ansatz, der auf der sozialwissenschaftlichen Netzwerkanalyse basiert sowie einem qualitativen Ansatz, in dem die „Inhalte sozialer Netzwerke, ihre Funktionsweise und soziale Rolle“ erforscht werden. ${ }^{447}$ Letzterer verwendet - aus Sicht der sozialwissenschaftlich orientierten Forschenden - den Netzwerkbegriff rein metaphorisch. 448 Ähnlich handhaben es Martin Stuber, Stefan Hächler und Hubert Steinke. Sie betrachten ein Netz als „meist nicht mehr als ein Geflecht von Beziehungen zwischen Akteuren mit unterschiedlichen und wechselseitig voneinander abhängigen Interessen [...].“449 Der hier benutzte Netzwerkbegriff wird nicht rein metaphorisch verwendet. Er bezieht sich auf ein Geflecht unterschiedlicher Akteurinnen und Akteure sowie Elemente, die relational Buchpraktiken ausführen und die dann, wenn sie dies tun, ein Kompilationsnetzwerk bilden.

Die Elemente des Kompilationsnetzwerkes führen Buchpraktiken aus oder wirken auf diese ein. Dies durch Menschen, die miteinander verbunden sind, wobei diese Handlungen durch „non-human entitites“ beeinflusst und gesteuert sein können. Die Buchpraktiken sind Teil einer übergeordneten, kontextabhängigen und sich verändernden Struktur und beinhalten unterschiedliche agentische Orientierungen. Sie können im Sinne von Emirbayer und Mische iterational, projektiv und praktisch-evaluativ sein, wobei auch der räumliche Aspekt von Bedeutung ist. Letzterer spielt sich auf einer anderen Ebene als die anderen Orientierungsweisen ab. Ausgeführt werden die Buchpraktiken von Personen, die miteinander in Verbindung stehen. Diese Beziehungen bilden die Basis eines Kompilationsnetzwerks. Dies gilt auch für die Weckerschen Kompilationsnetz-

447 Danuser, Regina; Hächler, Stefan; Kempe, Michael u. a.: Einleitung, in: Danuser, Regina/ Hächler Stefan; Kempe Michael; Mauelshagen Franz; Stuber Martin (Hg.): Wissen im Netz. Botanik und Pflanzentransfer in europäischen Korrespondenznetzen des 18. Jahrhunderts (= Colloquia Augustana). Berlin: De Gruyter, 2008, S. 9-28, hier S. 20.

448 Ebd. Zur sozialwissenschaftlichen Netzwerkanalyse siehe z. B. Wasserman, Stanley; Faust, Katherine: Social Network Analysis: Methods and Applications (= Structural Analysis in the Social Sciences). Cambridge: Cambridge University Press, 2004; Jansen, Dorothea: Einführung in die Netzwerkanalyse. Grundlagen, Methoden, Forschungsbeispiele. Wiesbaden: VS Verl. für Sozialwiss, 200633 Latour: On Actor-Network Theory. A few Clarifications, 1996. Vorreiter derselben war Ders.: Science in Action, 1987. Zum metaphorischen Gebrauch des Netzwerk-Begriffs siehe Roche, Daniel: Avant-propos. Réseaux des pouvoirs. Pouvoirs des réseaux dans l'Europe des Lumières, in: Beaurepaire, Pierre-Yves (Hg.): La plume et la toile. Pouvoirs et réseaux de correspondance dans l'Europe des Lumières (= Histoire). Arras: Artois presses université, 2002, S. 7-24.

449 Stuber, Martin; Hächler, Stefan; Steinke, Hubert: Teil I. Albrecht von Hallers Korrespondenznetz. Eine Gesamtanalyse, in: Stuber, Martin; Hächler, Stefan; Lienhard, Luc (Hg.): Hallers Netz. Ein europäischer Gelehrtenbriefwechsel zur Zeit der Aufklärung. Basel: Schwabe, 2005, S. 3-216, hier S. 18. 
werke, bei denen soziale Beziehungen die Voraussetzung für eine erfolgreiche Publikationstätigkeit bildeten. ${ }^{450}$ Aufgrund der grossen Bedeutung derselben für die Weckersche Textproduktion wird im Folgenden auf sein soziales Netzwerk eingegangen.

\subsection{Das soziale Netzwerk Johann Jacob Weckers}

Das soziale Netzwerk Johann Jacob Weckers ist nicht erforscht und aufgrund der Quellen auch nur zu Teilen rekonstruierbar. ${ }^{451}$ Dies anhand von Briefen und wenigen anderen zeitgenössischen Dokumenten. ${ }^{452}$ Gerade die Personen, die nicht direkt mit den Büchern zu tun hatten - also etwa die Botinnen und Boten sind nicht greifbar. Dennoch erhalten wir anhand der Quellen einen Einblick in sein in die Buchproduktion involviertes Netzwerk. ${ }^{453}$

Zum Weckerschen sozialen Netzwerk zählte zuvorderst Theodor Zwinger. ${ }^{454}$ Er war in unterschiedlichsten Funktionen an den Publikationen Johann Jacob Weckers beteiligt: Er korrigierte sie, scheint Vorreden geschrieben zu haben und erteilte Ratschläge. ${ }^{455}$ Im vermutlich ersten von Wecker überlieferten Brief aus dem Jahr 1571 - einige Briefe sind nicht datiert -, bedankte sich Wecker bei Zwinger für dessen Unterstützung. Er schreibt, dass er sich ihm gegenüber schon lange hätte dankbar erweisen müssen, da ihm der Doktor vor langem, während

\footnotetext{
450 Soziale Netzwerke waren nicht nur für die Weckersche Buchproduktion bedeutsam, sondern auch für die Herstellung anderer Books of Secrets, im Kontext der Paracelsistica sowie bei humanistischen Buchproduktionen. Kavey: Books of Secrets, 2007, chapter one; Neumann: Wissenspolitik in der frühen Neuzeit am Beispiel des Paracelsismus, 2011, S. 281; Hirstein, James: Wolfgang Capito and the other Docti in Johann Froben's Basel Print Shop, in: Rummel, Erika; Kooistra, Milton (Hg.): Reformation Sources. The Letters of Wolfgang Capito and his Fellow Reformers in Alsace and Switzerland (= Publications of the Centre for Reformation and Renaissance Studies. Essays and Studies). Toronto: Centre for Reformation and Renaissance Studies, 2007, S. 19-45; Kooistra: To Print or Not to Print, 2014, S. 135 f.

451 Dieses Kapitel basiert zu grossen Teilen auf meiner Masterarbeit: Zweifel: ,Communitas epistolaria‘ am Oberrhein, 2012.

$452 \mathrm{Zu}$ den Quellen siehe Kapitel 1.1.

453 Ebd.

$454 \mathrm{Zu}$ biographischen Angaben zu Theodor Zwinger siehe Kapitel 1.1.

455 Dazu siehe Kühlmann, Telle: Corpus Paracelsisticum: Band II, S. 729-731, 746. Eine Bitte Weckers, dass Zwinger eine Vorrede für ihn schreiben soll, findet sich in: Wecker, Johann Jacob: Brief an Theodor Zwinger. UB Basel, Frey-Gryn Mscr II 23:Nr. 487. Nicht nur Wecker, auch andere Personen der Basler Buchproduktion - insbesondere die Humanisten - tauschten sich aus und holten bei Freunden Expertise ein. Kooistra: To Print or Not to Print, 2014, S. 136 f.
} 
seiner Krankheit, „[...] vil liebs vnd gůts beweysen““. ${ }^{456}$ Deshalb habe er ihm Elsässer Wein schenken wollen; aufgrund der schlechten Jahre für den Wein sei dies jedoch nicht geschehen. Deshalb gebe er ihm ein kleines Fässlein gesottenen Muskat, den er doch mit seiner lieben Frau trinken möge. ${ }^{457}$ Aus diesem Brief geht hervor, dass die beiden Männer eine soziale Beziehung verband, die über Geschenke aufrechterhalten wurde. ${ }^{458}$ Gaben konnten dazu dienen, Beziehungen aufzubauen und beizubehalten: Sie waren ,a living medium of social action. “459 Damit kann der Gabentausch als eine Praktik verstanden werden, die unterschiedliche Akteurinnen und Akteure miteinander verbindet und kann dement-

456 Wecker, Johann Jacob: Brief an Theodor Zwinger. UB Basel, Frey-Gryn Mscr I 13:Bl.23.

457 „[...]. Dieweil ich aber mir <vor> langest <fürgenummen>, den herren doctor mit einem trincklin Elsesser wein zů verehren, fürgenummen, vnd aber sollichs von wegen der vngůten iaren, die mir bißher gehapt, nit hatt megen beschechen, biß zů diser zeit, do wol etwaß gůtz, aber wenig von Gott $\{v n ß\}$ beschert worden, schick ich euch auch wenig vnd gůt als ich hoff, namlich ein klein veßlin mit gesotten muscatel, bei meines schwagers fưr mitt einem solchen [unles.] verzeichnet, bittende wellen den selbigen nit verschmachen, sonder auff dißmal für gưt auff nemmen vnd in sampt euwer lieben hausfrauwen von vnsert wegen auß trincken. [...]“. Ebd.

458 Geschenke werden auch in anderen Briefen verzeichnet, so etwa in Wecker, Johann Jacob: Brief an Theodor Zwinger. UB Basel, Frey-Gryn Mscr II 28:Nr. 380. Neben Geschenken als Dank für die Arbeit, werden auch Neujahrsgeschenke erwähnt. Siehe ebd.; Ders.: Brief an Theodor Zwinger. UB Basel, Frey-Gryn Mscr II 28:Nr. 371; Ders.: Brief an Theodor Zwinger. UB Basel, Frey-Gryn Mscr II 4:Nr. 377. Zu Geschenken, insbesondere in der Frühen Neuzeit, u. a. Althoff, Gerd; StollbergRilinger, Barbara: Die Sprache der Gaben. Zu Logik und Semantik des Gabentauschs im vormodernen Europa, in: Jahrbücher für Geschichte Osteuropas 63 (1), 2015, S. 1-22; Grünbart, Michael (Hg.): Geschenke erhalten die Freundschaft. Gabentausch und Netzwerkpflege im europäischen Mittelalter; Akten des internationalen Kolloquiums Münster, 19.-20. November 2009 (= Byzantinistische Studien und Texte, Bd. 1). Berlin, Münster: LIT, 2011; Algazi, Gadi; Groebner, Valentin; Jussen, Bernhard (Hg.): Negotiating the Gift: Pre-modern Figurations of Exchange. Göttingen: Isd, 2003; Davis, Natalie Zemon: The Gift in Sixteenth-Century France (= Curti Lectures). Madison: The University of Wisconsin Press, 2000; Ben-Amos, Ilana K.: The Culture of Giving. Informal Support and Gift-exchange in Early Modern England (= Cambridge Social and Cultural Histories, 12). Cambridge: Cambridge University Press, 2008; Groebner, Valentin: Liebesgaben. Zu Geschenken, Freiwilligkeit und Abhängigkeit zwischen dem 14. und dem 16. Jahrhundert, in: Traverse. Zeitschrift für Geschichte 9, 2002, S. 39-52; Häberlein, Mark; Jeggle, Christof (Hg.): Materielle Grundlagen der Diplomatie. Schenken, Sammeln und Verhandeln in Spätmittelalter und Früher Neuzeit (= Irseer Schriften, N.F., 9). Konstanz: UVK-Verl.-Ges, 2013; Kettering, Sharon: Gift-Giving and Patronage in Early Modern France, in: French History 2, 1988, S. 131-151. Nach Gerd Althoff und Barbara Stollberg-Rilinger (Althoff, Stollberg-Rilinger: Die Sprache der Gaben, 2015, S. 1) „Klassisch und noch immer grundlegend“: Mauss, Marcel: Essai sur le don. Forme et raison de l'échange dans les sociétés archaïques, in: L’Année Sociologique 1 (1923/24), 1925, S. 30 -186. 459 Algazi, Groebner, Jussen: Negotiating the Gift, 2003, S. 12. Zu Geschenken als Medien sozialer Beziehungen siehe Groebner: Liebesgaben, 2002, S. 49; Althoff, Stollberg-Rilinger: Die Sprache der Gaben, 2015, S. 1. 
sprechend als Teil des Kompilationsnetzwerks verstanden werden, das alle Praktiken, die indirekt oder direkt zum Buch führten, miteinbezieht.

Bei diesen Gaben musste es sich nicht zwingend um Waren handeln. Als „Gegengeschenk“ könnten die Arbeiten Zwingers im Zusammenhang mit Weckers Publikationen betrachtet werden. Damit könnten die Weingeschenke Weckers auch als eine Art Lohn verstanden werden. ${ }^{460}$ Denn Universitätsprofessoren verdienten im späten 16. Jahrhundert nicht allzu gut, so dass unter anderem auch Medizinprofessoren die Möglichkeit nutzten, „sich durch nebenamtliche Berufstätigkeit Zuschüsse zu verschaffen.“" ${ }^{461}$ Darunter könnte beispielsweise das Verfassen von Vorreden sowie weiteren unterstützenden Arbeiten für die Weckersche Buchpublikation gefallen sein. ${ }^{462}$ So schrieb Wecker 1575 :

Indessen werde ich sowohl im Öffentlichen als auch im Privaten täglich von Arbeit überschüttet, und sei zu wenig geübt im Schreiben, und auch frage ich Dich immer wieder, damit $\mathrm{Du}$ in meinem Namen irgendeine Vorrede, oder einen Widmungsbrief erstellst und den neuen Titel und für das Werk setzt. ${ }^{463}$

Und ein Jahr später, wohl nach Zwingers Verfassen einer solchen Vorrede, schrieb Wecker: „Die prefation hab ich gesechen, gefelt mir vast wol, ist herlich vnd wolgemacht, wils aůff kürtzest verdienen." 464 Zwinger scheint nicht nur Vorreden für Wecker geschrieben zu haben, er knüpfte auch Verbindungen $\mathrm{zu}$ anderen Akteuren, wie etwa zu Perna. ${ }^{465}$ Diesbezüglich schrieb Wecker 1580:

Ehrenfester hochgelehrter Herr und Schwager, [...] euren Vertrag mit Pietro Perna mich betreffend lass ich mir ganz wohl gefallen. Ich bedanke mich aus diesem Grund sehr, dass ihr mir in dieser Sache so gutwillig gedient \{habt\} [und, SZ] will es bei nächster Gelegenheit

$460 \mathrm{Zu}$ Geschenken als Lohn: Groebner: Liebesgaben, 2002.

461 Bonjour, Edgar: Die Universität Basel von den Anfängen bis zur Gegenwart 1460-1960. Basel: Helbing u. Lichtenhahn, 1960, S. 61.

462 Siehe Kühlmann, Telle: Corpus Paracelsisticum: Band II, S. 729-731, 746; Wecker, Johann Jacob: Brief an Theodor Zwinger. UB Basel, Frey-Gryn Mscr II 4:Nr. 323; Ders.: Brief an Theodor Zwinger. UB Basel, Frey-Gryn Mscr I 4:Bl.71.

463 „Verum cum ego tum publicis tum priuatis negotijs obruor quotidie, parumque in scribendo sim exercitatus, ut meo nomine praefationem aliquam, uel epistolam dedicatoriam, construas titulumque operi nouum <operi> indas, te etiam atque etiam rogo.“ Wecker, Johann Jacob: Brief an Theodor Zwinger. UB Basel, Frey-Gryn Mscr II 4:Nr. 323.

464 Ders.: Brief an Theodor Zwinger. UB Basel, Frey-Gryn Mscr I 4:Bl.71.

$465 \mathrm{Zu}$ Pietro Perna und seiner Zusammenarbeit mit Wecker siehe insbesondere Kapitel 1.1 und 4.6. 
wiederum Euch und den anderen Herren, die bei dem Vertrag behilflich gewesen sind, vergelten und verdienen. ${ }^{466}$

In diesem Briefausschnitt ging es um einen „Vertrag“, den Zwinger Wecker vermittelte. Was dieser genau beinhaltete, kann aufgrund fehlender Quellen nicht beurteilt werden. Klar ist aber, dass Zwinger eine Vermittlerrolle zwischen Drucker und Kompilator zugewiesen werden kann. Zwinger war jedoch nicht der einzige Unterstützer Weckers, wie es dieses Zitat auch deutlich macht. Welche ,anderen herren" hier gemeint sind, lässt sich jedoch nicht mehr rekonstruieren. Doch auch sie sind aufgrund ihrer Vermittlerrolle Teil des Weckerschen Kompilationsnetzwerks. ${ }^{467}$

Wecker scheint auch für Zwinger gearbeitet zu haben. Darauf deutet folgender Briefausschnitt aus dem Jahre 1578 hin:

[...], hab disen winter (dieweil ich wenig zů schaffen, [...]) Organum ortis Rhetoricæ, vnd Dialecticæ methodicam, secum[?] tabularum formam gebracht, hoff die < die> selbigen in wenig tagen zů voll enden; [...]. So der herr ettwaß ein arbeit wüste, in arte Medica, so für eůch were, in tabulas zů redigieren, welte ich solchen rhatt mit danck an nemmen. Dan ich ein sonderen lůst zů Tabulis. ${ }^{468}$

In diesem Brief schrieb er, dass er die Tabellen für das Organum Logicum hergestellt habe und hoffe, diese in wenigen Tagen fertig zu stellen. ${ }^{469}$ Zudem bat er Wecker, ihn darüber zu informieren, falls er für ihn Tafeln redigieren, also herstellen könne, da er „ein sonderen lůst zů Tabulis“ habe. ${ }^{470}$ Diese Aufforderung deutet darauf hin, dass Zwinger nicht nur für Wecker, sondern Wecker auch für Zwinger gearbeitet hat. Es ist gar vorstellbar, dass er auch an den Tabellen des

466 „Erenůester hochgelerter herr vnd schwager, [...] euweren vertrag mitt Petro Perna von meinet wegen laß ich mir ganz woll gefallen. Sag eůch deshalben grosen danck, daß ir mir in diser sach so gůtwillig gedienett \{haben\} will eß bei nechster gelegenheitt widerumb vmb eůch sampt anderen herren, so bei dem vertrag behülfflich gwesen, beschůlden vnd verdienen.“ Wecker, Johann Jacob: Brief an Theodor Zwinger. UB Basel, Frey-Gryn Mscr II 27:Nr. 245.

467 Zum Kompilationsnetzwerk siehe Kapitel 3.

468 Wecker, Johann Jacob: Brief an Theodor Zwinger. UB Basel, Frey-Gryn Mscr II 28:Nr. 380. 469 Ders.: Organum Logicum. Ex Aristotele potissimum, alijsque uarijs authoribus collectum, in Tabularum formam redactum, ac methodicè digestum, [...]. Basileae: Per Evsebivm Episcop. \& Nicol. frat. hæredes, 1581.

470 Ders.: Brief an Theodor Zwinger. UB Basel, Frey-Gryn Mscr II 28:Nr. 380. 
Theatrum vitae humanae beteiligt war, da die gleiche Art Arbeit hinter der Produktion dieser und der Weckerschen Tabellen steckte. ${ }^{471}$

Auf der Ebene der Wissensorganisation von grosser Bedeutung war Simon Grynæus. ${ }^{472}$ Grynæus war häufig in Frankfurt unterwegs, weshalb Wecker immer wieder Bücher bei ihm bestellte: „,...] manglen mir noch etliche büecher, so D. Grineus mir zů schicken soll von Frankfurt, welche man zů Basel nitt bekummen mag“, schrieb er 1576 an Theodor Zwinger. ${ }^{473}$ Damit war Grynæus das Verbindungsglied im Weckerschen Kompilationsnetzwerk zur Frankfurter Buchmesse. ${ }^{474}$ Diese Verbindung war von grosser Wichtigkeit, da nur durch diese viele Bücher direkt aus Frankfurt beschafft werden konnten. Auch die Buchmesse selbst hatte eine bedeutende Funktion im Kompilationsnetzwerk inne: sie beeinflusste die endgültige Form dadurch, dass die Bücher zur Messe erscheinen sollten; aufgrund des Zeitdrucks blieb manchmal das eine oder andere Detail aussen vor.

471 Zwinger, Lykosthenes: Theatrum vitae humanae, 1565. Zu Tabellen bei Zwinger: Zedelmaier: Navigieren im Textuniversum, 2008, S. 122; Blair: Too Much to Know, 2010, S. 144-152. Zu solchen - ramistischen - Tabellen siehe auch Kapitel 4.4.

472 Möglicherweise ist hier Simon Grynæus der Jüngere (1539-1582) gemeint, der in Basel und Heidelberg studierte und 1564 zum Professor der Mathematik in Heidelberg ernannt wurde. Während seiner Zeit als Professor studierte er Medizin und promovierte 1568 in diesem Fach in Heidelberg. 1580 wurde er zum Professor für Ethik an der Universität Basel berufen, wo er zwei Jahre später an der Pest verstarb. Gantenbein, Urs Leo: Art. „Grynaeus, Simon“, in: Historisches Lexikon der Schweiz. Online unter: https://hls-dhs-dss.ch/de/articles/028659/2007-03-06/ [23.02. 2021]. Vom Alter her weniger wahrscheinlich, vom Kontext aber wahrscheinlicher ist die Zuweisung des Namens zu Simon Grynæus (1571-1621), dem Sohn des Samuel Grynæus und der Elisabeth Peyer (1531-1576). Letztere war die Witwe des Buchdruckers Nicolaus Bischoff (15311565), dem Sohn des Buchdruckers Nicolaus Bischoff (1501-1564), der 1518 an der Universität Basel immatrikuliert war und der zusammen mit Hieronymus Froben (1501-1563) die Druckerei Frobenius \& Episcopius gegründet hatte, die äusserst erfolgreich war. Simon Grynæus unterrichtete am Gymnasium und heiratete 1592 Anna Irmi. Siehe Fuchs-Eckert, Hans Peter: Art. „Bischoff“, in: Historisches Lexikon der Schweiz. Online unter: https://hls-dhs-dss.ch/de/articles/ 021016/2002-10-31 [23.02.2021]; Stroux, Ulrich: Auszug Stamm Grynaeus. Frühe Generationen. Online unter: https://www.stroux.org/patriz_f/stQV_f/GrA_f.pdf [23.02.2021]; Ders.: Auszug Stamm Grynaeus. Online unter: https://www.stroux.org/patriz_f/stQV_f/Gr_f.pdf [23.02.2021]; Ders.: Auszug Stamm Peyer. Online unter: http://www.stroux.org/patriz_f/stQV_f/Py_f.pdf [23.02. 2021]; Ders.: Auszug Stamm Bischoff. Nicolaus Bischoff oo Justina Froben $1501 \mathrm{ff}$. Online unter: https://www.stroux.org/patriz_f/stQV_f/BiB_f.pdf pdf [23.02.2021]. Diese Ausführungen basieren auf Zweifel: ,Communitas epistolaria‘ am Oberrhein, 2012, Glossar.

473 Wecker, Johann Jacob: Brief an Theodor Zwinger. UB Basel, Frey-Gryn Mscr II 28:Nr. 374. Einen weiteren Hinweis auf die Beschaffung von Büchern durch Grynæus findet sich in: Ders.: Brief an Theodor Zwinger. UB Basel, Frey-Gryn Mscr II 28:Nr. 376.

474 Siehe dazu Kapitel 3.3. 
Ein wichtiger Akteur beim letzten Schritt, das Buch „ad prelum“, also zum Druck, zu bringen, war der Drucker. Wecker arbeitete bei den De Secretis mit Pietro Perna zusammen. ${ }^{475}$ Diese Zusammenarbeit wurde höchst wahrscheinlich durch Theodor Zwinger hergestellt und war nicht immer konfliktfrei, wie wir später sehen werden. ${ }^{476}$ Neben ihm wurde auch bei Eusebius Episcopius gedruckt; auch in die Verhandlungen mit diesem Drucker war Theodor Zwinger teilweise involviert. $^{477}$

Ebenfalls Teil des Weckerschen Kompilationsnetzwerks war seine Frau, Anna Wecker. Sie wird immer wieder erwähnt in der Korrespondenz, unter anderem wegen einer Krankheit: „Meiner haußfrauwen kranckheitt verhindert mich seer vil, nitt allein an disem, sonder vil mher an der practic, ist nach on vnderlaß kranck, Gott welle ein mal ein vermügen haben“, heisst es in einem Brief aus dem Jahr 1573.478 Anna Weckers Erkrankung führte demnach dazu, dass Wecker weniger Zeit für die Publikationen, aber auch für seine medizinische Praxis hatte. Dies wiederum konnte sich auf die Inhalte auswirken, etwa dadurch, dass er weniger Zeit hatte, Bücher durchzuarbeiten, denen er Wissen entnahm. Seine Ehefrau war jedoch nicht nur passiv in die Weckersche Buchproduktion involviert: sie war als Botin direkt an der Korrespondenz beteiligt und übernahm nach dessen Tod die Rolle Johann Jacob Weckers in der 1588 erschienenen Ausgabe des Antidotarium speciale. ${ }^{479}$ Anna Wecker hatte eine Tochter, Katharina, aus ihrer

$475 \mathrm{Zu}$ Perna siehe ebd.

476 Siehe dazu Kapitel 4.6.

477 Siehe z. B. Wecker, Johann Jacob: Brief an Theodor Zwinger. UB Basel, Frey-Gryn Mscr II 28: Nr. 373. Da Nicolaus Bischoff (1501-1564) sowie dessen Sohn Nicolaus Episcopius d. J. (1531-1565) zum Zeitpunkt der Weckerschen Korrespondenz, die sich auf die 1570er und 1580er Jahre beschränkt, bereits verstorben waren, wird es sich hier um einen weiteren Sohn, Eusebius (1540 1599), handeln, der damals mit den Erben des Nicolaus Episcopius d. J. zusammenarbeitete. Von 1564 bis 1565, bis zum Tod des Nicolaus, hatten die Brüder die Druckerei gemeinsam betrieben. Reske: Die Buchdrucker des 16. und 17. Jahrhunderts im deutschen Sprachgebiet, 2015, S. 90. Eusebius Episcopius führte nicht nur eine Druckerei, sondern in Courcelles-les-Montbéliard auch eine Papiermühle, die durch Mitarbeitende betrieben wurde. Siehe Wilhelmi, Thomas: Ein Druckort mit besonderen Voraussetzungen. Das Mömpelgarder Buchwesen im späten 16. und 17. Jahrhundert, in: Lorenz, Sönke; Rückert, Peter (Hg.): Württemberg und Mömpelgard 600 Jahre Begegnung. Beiträge zur wissenschaftlichen Tagung vom 17. bis 19. September $1997 \mathrm{im}$ Hauptstaatsarchiv Stuttgart = Montbéliard - Wurtemberg, 600 Ans de Relations (= Schriften zur südwestdeutschen Landeskunde). Leinfelden-Echterdingen: DRW-Verlag, 1999, S. 303-311, hier S. $307 \mathrm{f}$. Zu Episcopius siehe auch Fuchs-Eckert: Art. „Bischoff“. In: Historisches Lexikon der Schweiz. Online unter: https://hls-dhs-dss.ch/de/articles/021016/2002-10-31/ [23.02.2021].

478 Wecker, Johann Jacob: Brief an Theodor Zwinger. UB Basel, Frey-Gryn Mscr II 28:Nr. 371. 479 Ders.: Brief an Theodor Zwinger. UB Basel, Frey-Gryn Mscr II 28:Nr. 378; Ders.: Antidotarium speciale, 1588. 
ersten Ehe mit Israel Aeschenberger, dem Stadtschreiber von Altdorf bei Nürnberg. Katharina ging 1572 mit dem Mediziner und Philosophen Nikolaus Taurellus (1547-1606) den Bund der Ehe ein. ${ }^{480}$ Letzterer war in das Weckersche Netzwerk integriert. Dies dadurch, dass er die ersten Kapitel der De Secretis libri XVII verfasste. ${ }^{481}$ Folglich war die Familie ein wichtiger Bestandteil des sozialen Geflechts und auch vieler Weckerscher Kompilationsnetzwerke.

In einige dieser Geflechte eingebunden war auch Lazarus von Schwendi (1522-1583). Er war Reichsfreiherr von Hohenlandsberg und - zur Zeit der We-

480 Classen: Der Liebes- und Ehediskurs vom hohen Mittelalter bis zum frühen 17. Jahrhundert, 2005, S. 295. Siehe auch: Wiswe, Hans: Kulturgeschichte der Kochkunst. Kochbücher und Rezepte aus zwei Jahrtausenden. München: Moos, 1970, S. 48; [Anonym]: Art. „Weckerin, (Anna)“, in: Zedler. Johann Heinrich Zendlers Grosses vollständiges Universallexicon aller Wissenschafften und Künste, Band 53, Leipzig/Halle: Johann Heinrich Zedler, 1747, S. 899, Sp. 1772; [Anonym]: Art. „Weggerin, Anna“, in: Amaranthes. Nutzbares, galantes und curiöses Frauenzimmer-Lexicon. [...], Leipzig 1715, Sp. 2106. Online unter: http://diglib.hab.de/drucke/ae-12/start.htm?image= 01075 [23.02.2021]. Nikolaus Taurellus (Oechslein) kam 1547 in Mömpelgard (Montbéliard) als Sohn eines protestantischen Stadtschreibers zur Welt. Seine Ausbildung führte ihn nach Tübingen und nach Basel, wo er sich mit der Theologie und der Medizin beschäftigte. 1565 erlangte er den Magistertitel in der Philosophie, fünf Jahre später den Doktortitel der Medizin. Ab 1572 unterrichtete er an der Universität Basel Medizin. Vermutlich 1577 folgte er auf die Ethikprofessur, die bis anhin Theodor Zwinger innegehabt hatte. Vor 1578 heiratete er Katharina Aeschenberger, die Tochter des Israel Aeschenberger und der Anna Keller. Gemeinsam hatten sie 13 Kinder. 1580 wurde Taurellus nach Altdorf an die Academia Norica berufen, wo er als Professor der Medizin wirkte. Nach dem Tod von Katharina Aeschenberger im Jahr 1598 oder 1599 heiratete Taurellus Ursula Haller von Hallerstein. Am 28. September 1606 verstarb Taurellus in Altdorf bei Nürnberg an der Pest.Vgl. Will, Georg Andreas: Nürnbergisches Gelehrten-Lexicon: oder Beschreibung aller Nürnbergischen Gelehrten beyderley Geschlechtes nach ihrem Leben, Veriensten und Schriften. Nürnberg, Altdorf: Lorenz Schüpfel, 1755-1758, S. 8; Groos, Karl: Art. „Taurellus, Nicolaus“, in: Allgemeine Deutsche Biographie 37 (1894), S. 467-471. Online unter: https://www.deutsche-bio graphie.de/pnd118801430.html\#adbcontent [23.02.2021]; Herbert, Jaumann: „Art. Taurellus, Nikolaus“, in: Neue Deutsche Biographie 25 (2013), S. 808-809. Online unter: https://www.deut sche-biographie.de/pnd118801430.html\#ndbcontent [23.02.2021]; Thomann, Günther. „Art. Taurellus, Nikolaus“; in: Biographisch-Bibliographisches Kirchenlexikon, Band XI. Hamm, Herzberg: Bautz, 1996, Sp. 574-579; Mährle, Wolfgang: Academica Norica. Wissenschaft und Bildung an der Nürnberger Hohen Schule in Altdorf (1575-1623). Stuttgart: Franz Steiner Verlag, 2000, S. 344, 400; Kühlmann, Telle: Corpus Paracelsisticum: Band II, S. 194; Wilhelm Gottlieb Tennemann nennt 1575 als das Jahr von Taurellus' Promotion. Tennemann, Wilhelm Gottlieb: Geschichte der Philosophie, Band 9, Teil 1. Leipzig: Johann Ambrosius Barth, 1814, S. 487. Siehe auch Wecker, Johann Jacob: Brief an Theodor Zwinger. UB Basel, Frey-Gryn Mscr II 28:Nr. 379. Diese Ausführungen basieren auf Zweifel: ,Communitas epistolaria‘ am Oberrhein, 2012, Glossar.

481 Wecker: De Secretis libri XVII, 1582b. 
ckerschen Publikationstätigkeit - ehemaliger Berater des Kaisers Maximilian II. ${ }^{482}$ Wecker scheint in den 1570er Jahren bis zu seinem Tod der Leibarzt von Schwendis gewesen zu sein. ${ }^{483}$ Darauf deutet die Korrespondenz hin, in der unter anderem ein Brief Weckers an Zwinger überliefert ist, in dem steht: „D. Schwendi leidet \{schon seit einigen Wochen\} schwer an der Fussgicht und der Gicht. “484 Wie die Beziehung zwischen den beiden zustande kam, kann nicht mehr rekonstruiert werden. Der erste Hinweis auf diese Verbindung findet sich in einem Brief Weckers an Theodor Zwinger aus dem Jahre 1572. Darin trat Wecker als Vermittler zwischen Theodor Zwinger und Lazarus von Schwendi hervor. ${ }^{485}$ Diese Rolle bestätigen weitere Briefe, in denen Wecker Zwinger im Namen von Schwendis immer

$482 \mathrm{Zu}$ Lazarus von Schwendi siehe u. a. Schlick, Hieronymus von: Das Tagebuch. Eine Selbstdarstellung aus den Jahren 1580 - 1582 (= Prameny k českým dějinám 16.-18. století : Ǩada B, Vita privata, Vol. 2). České Budějovice: Jihočeská Univ., Historicky Ústav Filozofické Fak, 2008; Greyerz, Kaspar von: Lazarus von Schwendi (1522-1583) and Late Humanism at Basel, in: Fleischer, Manfred Paul (Hg.): The Harvest of Humanism in Central Europe. Essays in honor of Lewis W. Spitz. St. Louis (Mo.) 1992, S.179-195; Greyerz, Kaspar von: Un moyenneur solitaire: Lazarus von Schwendi et la politique religieuse de l'Empire au XVIe siècle tardif, in: Arnold, Matthieu; Decot, Rolf (Hg.): Frömmigkeit und Spiritualität. Auswirkungen der Reformation im 16. und 17. Jahrhundert = Piété et spiritualité: l'impact de la Réformation aux XVIe et XVIIe siècles (= Veröffentlichungen des Instituts für Europäische Geschichte Mainz, Abteilung für Abendländische Religionsgeschichte. Beiheft). Mainz: P. von Zabern, 2002, S. 147-160; Neumann: Wissenspolitik in der frühen Neuzeit am Beispiel des Paracelsismus, passim; Schnur, Roman: Lazarus von Schwendi (1522-1583). Ein unerledigtes Thema der historischen Forschung, in: Zeitschrift für Historische Forschung 14, 1987, S. 27-46; Hepperle, Ingrid: Lazarus von Schwendi. Wie ein Schwabe am Oberrhein sein Glück machte. Ulm, Donau: Hess, 1997; Nicklas, Thomas: Um Macht und Einheit des Reiches: Konzeption und Wirklichkeit der Politik bei Lazarus von Schwendi (1522-1583). Husum: Matthiesen, 1995; Kluckhohn, August von: Art. „Schwendi, Lazarus Freiherr von“, in: Allgemeine Deutsche Biographie 33 (1891), S. 382-401. Online unter: https://www.deut sche-biographie.de/pnd118760130.html\#adbcontent [23.02.2021]; Nicklas, Thomas. Art. „Schwendi, Lazarus Freiherr von“, in: Neue Deutsche Biographie 24 (2010), S. 65f. Online unter: https://www.deutsche-biographie.de/sfz79756.html\#ndbcontent [23.02.2021]. Zu Kaiser Maximilian II. siehe u. a. Six, Barbara: Denkmal und Dynastie. König Maximilian II. auf dem Weg zu einem Bayerischen Nationalmuseum (= Miscellanea Bavarica Monacensia, 185). München: Herbert Utz, 2012; Fichtner, Paula Sutter: Emperor Maximilian II. New Haven: Yale University Press, 2001; Edelmayer, Friedrich; Kohler, Alfred (Hg.): Kaiser Maximilian II. Kultur und Politik im 16. Jahrhundert (= Wiener Beiträge zur Geschichte der Neuzeit, Bd. 19). Wien, München: Verl. für Geschichte und Politik; Oldenbourg, 1992.

483 Siehe dazu Schlick: Das Tagebuch, 2008, insbes. S. 284-289. Darauf, dass Wecker Schwendis Leibarzt war, deutet auch die Korrespondenz hin. Zur Korrespondenz siehe Kapitel 1.1. 484 „[...] D. Suendius chyragra et podagra \{iam per aliquot ebdomadas $\}$ grauiter laborat.“ Wecker, Johann Jacob: Brief an Theodor Zwinger. UB Basel, Frey-Gryn Mscr II 5:Nr. 97.

485 Ders.: Brief an Theodor Zwinger. UB Basel, Frey-Gryn Mscr II 4:Nr. 314. 
wieder darum bat, letzteren zu besuchen. ${ }^{486}$ Ein Hinweis darauf, dass Wecker für Schwendi tätig war, findet sich auch im Tagebuch des Pfalzgrafen Hieronymus Schlick, das dieser zwischen 1580 und 1582 verfasste. ${ }^{487}$ Dort wird ein „Hans Jacob“ im Zusammenhang mit Lazarus von Schwendi erwähnt: „Darnach hab ich brieff vom apotecker von Colmar, Hans Jacob, des von Schwenden cämmerling, und meinem herrn schwagern, dem von Schwenden, bekommen. “488 Den deutlichsten Beleg findet sich jedoch in einem Brief Weckers an Zwinger aus dem Jahr 1574, in dem es heisst: „Allein hatt mir D. Sůendis vor langest verheissen kaiserliche vnd burgerlicher schatzungen freÿheiten zů erlangen, [...] Burgerlicher schatzung bin ich freij, so lang ich am dienst bleib [...]. “489 Wecker war demnach, zumindest während seiner Dienstzeit, von den bürgerlichen Steuern befreit. ${ }^{490}$ Folglich war die Beziehung zwischen Wecker und von Schwendi nicht eine, die rein auf dem Papier bestand, sondern eine, bei der die entsprechenden Personen im Alltag miteinander verbunden waren. Schwendi war auch deshalb Teil des Weckerschen Kompilationsnetzwerks, weil er für die De Secretis libri XVII als Widmungsträger fungierte. ${ }^{491}$ Somit kam ihm eine zusätzliche Funktion in der Weckerschen Buchproduktion zu. ${ }^{492}$

Alle mir bekannten Personen des sozialen Netzwerks Johann Jacob Weckers sind damit benannt. Diese Aufzählung verdeutlicht, dass alle darin involvierten Personen auch in die Weckersche Publikationstätigkeit eingebunden waren. Es

486 Siehe u. a. Ders.: Brief an Theodor Zwinger. UB Basel, Frey-Gryn Mscr II 4:Nr. 315; Ders.: Brief an Theodor Zwinger. UB Basel, $\mathrm{G}^{2}$ II 8 fol. 180 (entspricht Dems.: Brief an Theodor Zwinger. UB Basel, Frey-Gryn Mscr I 12:Nr. 346.); Ders.: Brief an Theodor Zwinger. UB Basel, Frey-Gryn Mscr II 28:Nr. 377; Ders.: Brief an Theodor Zwinger. UB Basel, Frey-Gryn Mscr II 4:Nr. 329.

487 Hieronymus Schlick kam 1556 oder 1557 als Kind einer Adelsfamilie zur Welt. Er besuchte vermutlich die humanistische Schule in Joachimsthal und studierte später in Prag, Leipzig, Marburg und auf den Britischen Inseln. Im Dezember 1581 trat er eine Stelle als Hofprediger am Hof Johann Kasimirs von der Pfalz-Lautern (1543-1592) an, darauf folgte eine Tätigkeit als Fürstenrat des württembergischen Kurfürsten Ludwig (1568-1593). Schlick: Das Tagebuch, 2008, S. $26 \mathrm{f} .$, 37-40. Diese Ausführungen basieren auf Zweifel: ,Communitas epistolaria‘ am Oberrhein, 2012, S. 50 .

488 Schlick: Das Tagebuch, 2008, S. 290. Die Angaben zu „Hans Jacob“ finden sich auf den Seiten 284-290.

489 Wecker, Johann Jacob: Brief an Theodor Zwinger. UB Basel, Frey-Gryn Mscr II 5:Nr. 96. 490 Ebd.

491 Ders.: De Secretis libri XVII, 1582. Auch Michael Toxites (1514-1581) widmete an Lazarus von Schwendi siehe Neumann: Wissenspolitik in der frühen Neuzeit am Beispiel des Paracelsismus, 2011, S. 258, 271. Hans-Peter Neumann geht auch auf die Verbindung zwischen Toxites und von Schwendi ein und darauf, dass letzterer dem Paracelsismus durchaus wohlgesonnen war. Ebd., S. 259 f., 272.

492 Darauf wird im folgenden Kapitel zu Buchpraktiken eingegangen. 
sind keine sozialen Kontakte ohne Bezug zur Textproduktion überliefert. Dies zeigt, wie verflochten die frühneuzeitliche Kompilationsproduktion mit den sozialen Netzwerken ihrer Akteurinnen und Akteure war. Sie alle waren in die Buchpraktiken involviert, die zu den Weckerschen Kompilationen führten. Doch welche Buchpraktiken waren dies? Und wer war an diesen beteiligt? Diesen Fragen wird im folgenden Kapitel nachgegangen, das sich mit den Buchpraktiken der Weckerschen Kompilationsnetzwerke befasst. 